\title{
ON. ANALYTIC FAMILIES OF COMPACT RIEMANN SURFACES WITH NON-TRIVIAL AUTOMORPHISMS
}

\author{
AKIKAZU KURIBAYASHI
}

\section{Introduction}

The purpose of this paper is to investigate the moduli of compact Riemann surfaces with non-trivial automorphisms.

We shall explain our problems by giving a summary of the contents.

In $\S 1$, we shall explain by some examples from where our problems occur. We know that the problems of representations of the group of automorphisms as linear transformations of the spaces of differentials have been studied. Our first subject is a converse of these problems in a certain sense, that is, giving a group $G$ and a representation $S$ of $G$, we wish to find such a Riemann surface that has $G$ as a subgroup of the group of automorphisms, and where the representation of the group $G$ is equivalent to $S$. From here many interesting problems arise.

In $\S 2$, we consider a family $\Omega(G, \rho)$ of Riemann surfaces with a prescribed type of automorphisms (cf. 2.1) and study some properties of the family, by which we are able to obtain some information about the structures of compact Riemann surfaces of small genera, i.e., of genus 2 and 3 . It seems to us an interesting matter that we obtain Theorem 2.17 as a residual product.

In $\S 3$, we consider a family $\Omega\left(g^{\prime}, n,\left\{\nu_{1}, \ldots, \nu_{r}\right\}\right)$ of Riemann surfaces which is a subfamily of $\Omega(n, \rho)$ with respect to the exponents $\nu_{1}, \ldots, \nu_{r}$ subject to certain conditions (cf. 3.1) and following the methods of Teichmüller [19], Ahlfors [2], and Bers [3], we shall devote ourselves to construct from this family a complex manifold $\Lambda$, which we shall call a generalized Teichmüller space. The main result of this section is Theorem 3.21, which asserts that $\Lambda$ is a $3 g^{\prime}-3+r$ dimensional complex analytic manifold. Here the number $3 g^{\prime}-3+r$ is nothing but the number of linearly independent quadratic differentials which are invariant by the given automorphism with $r$ fixed points.

Received January 12, 1966. 
In $\S 4$, we shall deal with a special family $\Omega\left(0, n,\left\{\nu_{1}, \ldots, \nu_{r}\right\}\right)$, from which we obtain a generalized Teichmüller space $A\left(0, n,\left\{\nu_{i}\right\}\right)$. We see that Riemann surfaces $R$ belonging to $\Omega\left(0, n,\left\{\nu_{i}\right\}\right)$ are of the form

$$
y^{n}=\left(x-a_{1}\right)^{m_{1}} \cdots\left(x-a_{s}\right)^{m_{s}}, n+m_{1}+\cdots+m_{s}, r=s+1,
$$

where $m_{i}(i=1, \ldots, s)$ are the numbers which are completely determined by $\nu_{1}, \ldots, \nu_{r}$ as shown in Proposition 3.4.

Let $J$ be the jacobian variety of $R$, and let $\zeta_{0}$ be the birational correspondence of $R$ with itself given by $(x, y) \rightarrow(x, \zeta y)$, where $\zeta$ is a primitive $n$-th root of unity. Denote by $\theta(\zeta)$ the automorphism of $J$ corresponding to $\zeta_{0}$. Let $\mathscr{C}$ be the canonical polarization of $J$, and $\rho$ the automorphism of $Q(\zeta)$ such that $\zeta^{\rho}=\zeta^{-1}$. Then we get a polarized abelian variety of type $\{Q(\zeta), \phi, \rho\}$ in the sense of Shimura [16] for a certain representation $\phi$ of degree $g$. Here $g$ is the genus of $R$.

On the other hand it is known by Shimura [16] that there exists a symmetric domain $H$ which parametrizes an analytic family of polarized abelian varieties $\mathscr{P}$ of type $\{Q(\zeta), \phi, \rho\}$.

We shall investigate the appearances of the jacobian varieties in this family of abelian varieties. For this purpose we consider the question whether there exists a holomorphic mapping of the generalized Teichmüller space $A$ into the symmetric domain $H$. The answer is affirmative, as shown in Theorem 4.13 , which is one of our main theorems in the present paper.

In $\S 5$, we shall discuss the holomorphy of parameters in the above equation of Riemann surfaces with respect to the complex structure of the generalized Teichmüller space $A$. For that purpose we consider the moduli-variety $\tilde{\mathfrak{B}}$ for the family of abelian varieties introduced in [18], which is the quotient of $H$ by a discontinuous group $\Gamma$. Let $S=\left\{\left(x_{1}, \ldots, x_{s}\right) \in C^{s} ; x_{i} \neq x_{j}\right.$, if $\left.i \neq j\right\}$. Then we can let $\left(R_{x}, \sigma_{x}\right)$ correspond to each $\left(x_{1}, \ldots, x_{s}\right) \in S$. Let $\lambda$ be a point of $\Lambda$ corresponding to $\left(R_{x}, \sigma_{x}\right)$. Then we obtain a rational mapping $\psi: S \rightarrow \tilde{\mathfrak{B}}$ which relates, roughly speaking, the parameters $x_{1}, \ldots, x_{s}$ holomorphically to $\lambda$. The precise statement of this fact is given in Theorem 5.9, which is our second main theorem.

In $\S 6$, we consider, as an example, a special case of the family $\Omega(0,7,\{1$, $1,1,2\})$ and apply the theory developed in the above sections to it. In this 
case, we see that both $\Lambda$ and $H$ are one dimensional manifolds and the mapping of $A$ into $H$ is a ratio of the periods of the differentials of the first kind of the Riemann surfaces (cf. 6.2). As for the holomorphy of parameters we can construct an invariant which is holomorphic in the whole space $A$ (cf. Theorem 6.7).

The author should like to express his hearty thanks to Prof. G. Shimura who has led him to this investigation and has given many valuable advices and constant encouragements. And the author also expresses his sincere thanks to Prof. S. Koizumi and Prof. M. Kuga for their kind advices and hearty encouragements.

\section{$\S 1$. Preliminary consideration}

1.1. Let $R$ be a compact Riemann surface of genus $g$; we can express an arbitrary meromorphic differential on $R$ in the form $\omega=f d g$, where $f, g$ are meromorphic functions on $R$. Let $\sigma$ be a holomorphic mapping of another Riemann surface $R^{\prime}$ onto $R$. If we set $f_{1}(z)=f(\sigma(z)), g_{1}(z)=g(\sigma(z))$, then $f_{1}(z), g_{1}(z)$ are meromorphic functions on $R^{\prime}$, and $\omega_{1}=f_{1} d g_{1}$ is a meromorphic differential on $R^{\prime}$. We denote this differential by $\omega_{1}=\omega \sigma$. Particularly if $R=R^{\prime}$, and if $\sigma$ is an automorphism of $R$, then $\omega_{1}$ is a meromorphic differential on $R$. Moreover if $\omega$ is a differential of the first kind, then $\omega_{1}$ is also of the first kind, and $\left(\omega_{1}+\omega_{2}\right) \sigma=\omega_{1} \sigma+\omega_{2} \sigma,(\lambda \omega)_{\sigma}=\lambda(\omega \sigma)$ where $\lambda$ is a complex number. We shall denote by $V(R)$, or simply by $V$, the complex vector space of all differentials of the first kind on $R$. Let $G$ be a subgroup of the group of all automorphisms of $R$. Every element $\sigma$ of $G$ induces a linear mapping of $V$ onto $V$ as we have seen above and moreover we see that $\omega(\sigma \tau)=(\omega \sigma) \tau$ for arbitrary $\sigma, \tau \in G$. Therefore fixing a basis of $V$, we obtain a matrix representation $\rho$ of $G$. It is known that this representation is faithful if the genus of $R$ is greater than one, for a proof see [9, p. 416].

1.2. Lemma. The notation being as above, let $\bar{\rho}$ be the complex conjugate of $\rho$. Then the representation

$$
\left(\begin{array}{ll}
\rho & 0 \\
0 & \bar{\rho}
\end{array}\right)
$$

of $G$ is equivalent to a rational representation of $G$. Moreover, $\operatorname{tr} \rho(\sigma)+\operatorname{tr} \bar{\rho}(\sigma)$ is a rational integer for every $\sigma \in G$. 
The first assertion is classical and well-known. For a proof, see for example [7; pp 270-272]. The second assertion follows easily from the first assertion.

1.3. Let $R$ and $G$ be as above in 1.1. Let $R^{\prime}$ be the quotient of $R$ by $G$. Then $R^{\prime}$ has a structure of compact Riemann surface, and $R$ can be regarded as a Galois covering of $R^{\prime}$ whose Galois group is $G$.

Let $K$ (resp. $K^{\prime}$ ) be the field of meromorphic functions on $R$ (resp. $R^{\prime}$ ). Then $K$ is a Galois extension of $K^{\prime}$, whose Galois group can be identified with $G$ in a natural manner.

If $G$ is a cyclic group of order $n$, then $\rho$ can be determined by $\rho(\sigma)$ for a generator $\sigma$ of $G$. Furthermore we can transform $\rho$ into a diagonal form, i.e., we can find a basis $\left\{\omega_{1}, \ldots, \omega_{g}\right\}$ of $V$ so that

$$
\left(\begin{array}{c}
\omega_{1} \sigma \\
\cdot \\
\cdot \\
\cdot \\
\omega_{g} \sigma
\end{array}\right)=\left(\begin{array}{ccc}
\alpha_{1} & & 0 \\
& \cdot & \\
& \cdot & \\
& & \cdot \\
0 & & \alpha_{g}
\end{array}\right)\left(\begin{array}{c}
\omega_{1} \\
\cdot \\
\cdot \\
\cdot \\
\omega_{g}
\end{array}\right)
$$

with $n$-th roots of unity $\alpha_{1}, \ldots, \alpha_{g}$, which may not be primitive. Let $p$ be the number of indices $k$ for which $\alpha_{k}=1$. It is well-known that $p$ is equal to the genus of $R^{\prime}$. We shall denote sometimes the above matrix by $\left[\alpha_{1}, \ldots, \alpha_{g}\right]$.

1.4. As explained in the Introduction, our subject of study is a family of Riemann surfaces with non-trivial automorphisms. In this paper we shall consider exclusively the case where the group of automorphisms is a cyclic group of prime order, though one can certainly investigate a more general case.

Now we shall give some examples.

1.5. Example. Let $R$ be a Riemann surface defined by the equation

$$
y^{\tilde{T}}=\left(x-a_{1}\right)\left(x-a_{2}\right)\left(x-a_{3}\right) \quad\left(a_{i} \neq a_{j} \text { if } i \neq j\right) .
$$

We see easily that $R$ is of genus 6 , and $\left\{y^{-6} d x, y^{-6} x d x, y^{-5} d x, y^{-5} x d x, y^{-4} d x, y^{-3} d x\right\}$ provides a basis of $V$. A map $\sigma:(x, y) \rightarrow(x, c y)$ is an automorphism of $R$, where $\zeta$ is a primitive 7 -th root of unity. This $\sigma$ generates a cyclic group $G$ of order 7. If we write the above basis $\left\{\omega_{1}, \ldots, \omega_{6}\right\}$ in order, then we have $\left(1_{1} \sigma=\zeta \omega_{1}, \omega_{2} \sigma=\zeta \omega_{2}, \omega_{3} \sigma=\zeta^{2} \omega_{3}, \omega_{4} \sigma=\zeta^{2} \omega_{4}, \omega_{5} \sigma=\zeta^{3} \omega_{5}, \omega_{6} \sigma=\zeta^{4} \omega_{\mathrm{i}}\right.$. Therefore we can express these relation as follows : 


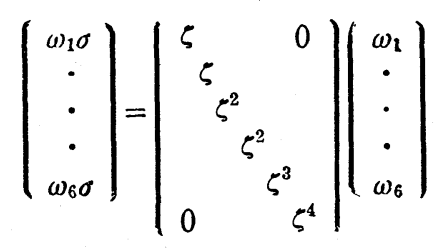

1.6. ExAmple. Let $R$ be the Riemann surface defined by the equation:

$$
y^{2}=\left(x-a_{1}\right)\left(x+a_{1}\right)\left(x-a_{2}\right)\left(x+a_{2}\right)\left(x-a_{3}\right)\left(x+a_{3}\right) \quad\left(a_{i} \neq a_{j}, \text { if } i \neq j\right) .
$$

It can be seen that $R$ is of genus 2 , and $\left\{y^{-1} d x, y^{-1} x d x\right\}$ is a basis of $V$. A map $\sigma:(x, y) \rightarrow(-x,-y)$ is an automorphism of $R$. This $\sigma$ generates a cyclic group $G$ of order 2 . If we set $\omega_{1}=y^{-1} d x, \omega_{2}=y^{-1} x d x$, then $\omega_{1} \sigma=\omega_{1}, \omega_{2} \sigma=-\omega_{2}$. Therefore we have

$$
\left(\begin{array}{c}
\omega_{1} \sigma \\
\omega_{2} \sigma
\end{array}\right)=\left(\begin{array}{cc}
1 & 0 \\
0 & -1
\end{array}\right)\left(\begin{array}{c}
\omega_{1} \\
\omega_{2}
\end{array}\right)
$$

Let us now recall the following well-known lemma from Galois theory.

1.7. Lemma. Let $K$ be a Galois extension of a field $k$. Suppose that the Galois group is cyclic and of order $n$, and $k$ contains a primitive $n$-th root of unity $\zeta$. Then for every generator of the Galois group, there exists an element $y$ of $K$ such that $\sigma(y)=5 y, K=k(y)$ and $y^{n} \in k$.

1.8. We shall apply this lemma to the fields of algebraic functions, which are considered in the above. Let $R, G, R^{\prime}, \rho, p, K, K^{\prime}$ be as in 1.3. Suppose that $G$ is a cyclic group whose order is a prime number $n$. Let $\sigma$ be a generator of the Galois group and let $\zeta=e^{2 \pi i / n}$.

(Case 1: $p=0$ ). Then $R^{\prime}$ is a Riemann sphere, and $K^{\prime}=C(x)$, where $C(x)$ is a rational function field. So we have

(1.8.1) $K=C(x, y), y^{n}=f(x), \sigma(x, y)=(x, \zeta y)$

where $f(x)$ is a polynomial in $x$. Therefore in general we can write the equation of the Riemann surface in the form

(1.8.2) $y^{n}=\left(x-a_{1}\right)^{m_{1}}\left(x-a_{2}\right)^{m_{2}} \cdots\left(x-a_{s}\right)^{m_{s}}$

with distinct complex numbers $a_{1}, a_{2}, \ldots, a_{s}$, and positive integers $m_{1}, m_{2}, \ldots, m_{s}$ less than $n$. Without loss of generality, we may assume that

(1.8.3) $n+m_{1}+m_{2}+\cdots+m_{s}$. 
In fact, assume that $m_{1}+m_{2}+\cdots+m_{s}=n q$ with an integer $q$. Let $x^{\prime}=\left(x-a_{1}\right)^{-1}$, $b_{i}=\left(a_{i}-a_{1}\right)^{-1},(i=2, \ldots, s)$ and $y^{\prime}=\left\{\left(a_{1}-a_{2}\right)^{m_{2}} \cdots\left(a_{1}-a_{s}\right)^{m_{s}}\right\}^{-1 / n}\left(x-a_{1}\right)^{-q} y$. Then we have $K=C\left(x^{\prime}, y^{\prime}\right), \sigma\left(x^{\prime}, y^{\prime}\right)=\left(x^{\prime}, \zeta y^{\prime}\right)$ and $y^{\prime n}=\left(x^{\prime}-b_{2}\right)^{m_{2}} \cdots\left(x^{\prime}-b_{s}\right)^{m_{s}}$ $n+m_{2}+m_{3}+\cdots+m_{s}$.

Now coming back to the original notation of (1.8.1) and (1.8.2) with the assumption (1.8.3), we obtain, by means of Riemann-Hurwitz formula,

(1.8.4) $2 g=(n-1)(s-1)$.

We can further normalize the equation (1.8.2) in the form

$$
y^{n}=x^{m_{1}}(x-1)^{m_{2}}\left(x-c_{1}\right)^{m_{2}} \cdots\left(x-c_{s-2}\right)^{m_{s}}
$$

with distinct complex numbers $c_{1}, \ldots, c_{s-2}$ which are different from 0 and 1 .

(Case 2: $p=1$ ). Then $K^{\prime}$ is an elliptic function field. Hence $K^{\prime}=C(u, v)$ where $u^{2}=4 v^{3}-\gamma_{2} v-\gamma_{3}, \gamma_{2}^{3}-27 \gamma_{3}^{2} \neq 0$. So we have

$$
K=C(u, v, y), y^{n}=a(v)+b(v) u, \sigma(u, v, y)=(u, v, \zeta y)
$$

where $a(v), b(v)$ are rational functions in $v$.

(Case $3: p=2$ ). Then $K^{\prime}$ is a hyperelliptic function field, and so $K^{\prime}=C(u, v)$ where $u^{2}=\prod_{i=1}^{5}\left(v-a_{i}\right)$ with distinct $a_{i}$. So we have

$$
\text { (1.8.7) } K=C(u, v, y), y^{n}=a(v)+b(v) u, \sigma(u, v, y)=(u, v, \zeta y)
$$

where $a(v), b(v)$ are rational functions in $v$.

Here we should notice that in each case, $G$ is generated by an automorphism $\sigma$ which transforms $y$ to $\zeta y$ and leaves all elements of $K^{\prime}$ invariant, where $\zeta=e^{2 \pi i / n}$.

1.9. Example. Let $G$ be a cyclic group of order 7 , and $\sigma$ a generator of $G$. We wish to determine a Riemann surface of genus 3 on which $\sigma$ operates as an automorphism so that, for a suitable basis $\omega_{1}, \omega_{2}, \omega_{3}$ of differentials of the first kind of it, one has

$$
\left(\begin{array}{c}
\omega_{1} \sigma \\
\omega_{2} \sigma \\
\omega_{3} \sigma
\end{array}\right)=\left(\begin{array}{cc}
\zeta & 0 \\
\zeta^{2} \\
0 & \zeta^{3}
\end{array}\right)\left(\begin{array}{l}
\omega_{1} \\
\omega_{2} \\
\omega_{3}
\end{array}\right), \quad \rho=\left[\zeta, \zeta^{2}, \zeta^{3}\right]=\left(\begin{array}{cc}
\zeta & 0 \\
\zeta^{2} \\
0 & \zeta^{3}
\end{array}\right),
$$

where $\zeta=e^{2 \pi i / 7}$. From the result of 1.8 , we see that such a Riemann surface must be defined by an equation of the form

$$
y^{7}=x^{m_{1}}(x-1)^{m_{2}}
$$


and the automorphism is given by $\sigma(x, y)=(x, \zeta y)$. Furthermore we see later that $m_{1}=1, m_{2}=1$, (or, $m_{1}=1, m_{2}=5$ ), (cf. 2.13).

1.10. Example. Let $G$ be a group of order 2, and $\sigma$ the generator of $G$. We wish to determine a Riemann surface $R$ of genus $g$ on which $\sigma$ operates as an automorphism so that, for a suitable basis $\omega_{1}, \ldots, \omega_{g}$ of $V(R)$, one has

$$
\left(\begin{array}{c}
\omega_{1} \sigma \\
\cdot \\
\cdot \\
\omega_{g} \sigma
\end{array}\right)=\left(\begin{array}{ccc}
-1 & & 0 \\
& \cdot & \\
& \cdot & \\
0 & & -1
\end{array}\right)\left(\begin{array}{c}
\omega_{1} \\
\cdot \\
\cdot \\
\omega_{g}
\end{array}\right), \rho=\left(\begin{array}{ccc}
-1 & & 0 \\
& & \cdot \\
0 & & -1
\end{array}\right)=[-1, \ldots,-1] .
$$

By the result of 1.8 , such a Riemann surface should be determined by an equation:

$$
y^{2}=x(x-1)\left(x-a_{1}\right) \cdots\left(x-a_{2 g-1}\right)
$$

where $a_{1}, \ldots, a_{2 g-1}$ are different from each other and are not equal to 0,1 .

1.11. In general one can ask the question of finding Riemann surfaces with an automorphism whose representation by differentials of the first kind is equivalent to a given matrix $\rho$, as described in the above examples. With an arbitrary $\rho$, there may be no such Riemann surfaces. But for a suitable $\rho$ there may exist Riemann surfaces of prescribed type with several parameters as we see in Example 1.10. It may also happen that there is no continuous parameter for such Riemann surfaces, as in Example 1.9.

Our first problem is to investigate these phenomena in detail for compact Riemann surfaces of genus 2 and 3 . This will be treated in the next section.

Our second problem is to construct a complex manifold $A$ which parametrizes compact Riemann surfaces of genus $g(g \geqq 2)$ with a given type of automorphism. Such a manifold may be regarded as a generalization of Teichmüller space.

Our third problem is to clarify the relation between the following three kinds of parameters:

(1) the point on $\Lambda$ corresponding to a Riemann surface $R$.

(2) the periods of the integrals of the first kind on $R$.

(3) the parameters $a_{1}, \ldots, a_{s}$ in the equation (1.8.2). 


\section{§2. A family of Riemann surfaces: $\Omega(n, \rho)$}

2.1. Let $n$ be a prime number, $g$ a positive integer $>1$, and $\rho$ a complex matrix of degree $g$ such that $\rho^{n}=1$. Let us consider a couple $(R, \sigma)$ formed by a compact Riemann surface $R$ and an automorphism $\sigma$ of $R$ of order $n$ such that the representation of $\sigma$ in the vector space $V(R)$ of differentials of the first kind on $R$ is equivalent to $\rho$. We say that $(R, \sigma)$ and $\left(R^{\prime}, \sigma^{\prime}\right)$ are isomorphic if there exists a holomorphic bijection $f: R \rightarrow R^{\prime}$ such that $f_{\sigma}=\sigma^{\prime} f$. We denote by $\langle R, \sigma\rangle$ the isomorphism class of $(R, \sigma)$ with given $n$ and $\rho$, and we denote by $\Omega(n, \rho)$ the set of all classes $\langle R, \sigma\rangle$.

As an example if $G=\{1\}, \rho=I$, then $\Omega$ consists of all Riemann surfaces of genus $g$; that is, $\Omega$ is nothing but the so-called space of moduli of Riemann surfaces of genus $g$.

\subsection{Proposition.}

(1) $\Omega\left(n, \rho_{0}\right)=\phi$ if $\rho_{0}=[1, \ldots, 1], n \geqq 2$.

(2) $\Omega\left(n, \rho_{2}\right)=\phi$ if $\rho_{2}=\left[1,1, \zeta^{\lambda}\right], n \geqq 3, \zeta=e^{2 \pi i / n}, 1 \leqq \lambda \leqq n-1$.

Proof. The assertion (1) is well-known (cf. [9; p. 416]). But here we give a proof for both (1) and (2) by means of the formula of Riemann-Hurwitz:

$$
2 g-2=[G: 1]\left(2 g^{\prime}-2\right)+\sum_{i=1}^{r}\left(e_{i}-1\right),
$$

where $g$ is the genus of $R$ and $g^{\prime}$ the genus of $R^{\prime}=R / G$, and $e_{i}$ the ramification degrees.

(1) Since $g=g^{\prime}$, we must have $n=1$, which shows that $\Omega\left(n, \rho_{0}\right)=\phi$ for $n \geqq 2$.

(2) Since $g=3, g^{\prime}=2,[G: 1] \geqq 3$, the left hand side of the equation is equal to 4 , but the right hand side $\geqq 2 n$, which is a contradiction when $n \geqq 3$ (If $n=2, R$ must be an unramified covering of $R^{\prime}$ ).

2.3. Now we notice that every automorphism of $R$ can be represented also in the space $V^{*}=V^{*}(R)$ of regular quadratic differentials of $R$. It is wellknown that the dimension of $V^{*}$ is $3 g-3$. Let $\rho^{*}$ be a matrix of size $3 g-3$ such that $\rho^{* n}=1$.

We denote by $\Omega\left(n, \rho, \rho^{*}\right)$ the set of all isomorphism classes $\langle R, \sigma\rangle$ which satisfies the following conditions:

(1) $\langle R, \sigma\rangle$ represents a member of $\Omega(n, \rho)$. 
(2) The representation of $\sigma$ in the space $V^{*}(R)$ is equivalent to $\rho^{*}$.

2.4. Remark. If we put $g=2, n=2, \rho=[-1,-1], \rho^{*}=[1,1,1]$, then $\Omega\left(2, \rho, \rho^{*}\right)$ is the set of all $\langle R, \sigma\rangle$, where $R$ is a Riemann surface defined by the equation:

$$
y^{2}=x(x-1)\left(x-a_{1}\right)\left(x-a_{2}\right)\left(x-a_{3}\right)
$$

and $\sigma$ is an automorphism of $R$ which maps $x$ to $x$, and $y$ to $-y$.

In order to study the properties of $\rho, \rho^{*}$, we may use the trace formulas of Eichler $[7,8]$.

2.5. Lemma. Let $\sigma$ be an automorphism of $R$ of order $n$ and let $\zeta=e^{2 \pi i / n}$. We denote by $\operatorname{tr} \rho(\sigma)$ the trace of the representation of $\sigma$ in the space $V(R)$. Let $t$ be a local parameter at a point $P$ which is fixed by $\sigma$. Then o can be represented as $t \rightarrow \zeta^{\nu} t+\zeta^{\prime} t^{2}+\cdots$. Here $\nu$ is an integer such that $1 \leqq \nu<n-1$, and does not depend on the choice of local parameters. Then we have the formula:

$$
\operatorname{tr} \rho(\sigma)=1+\sum_{i=1}^{r} \frac{\zeta^{\nu_{i}}}{1-\zeta^{\nu_{i}}}
$$

where $r$ is the number of fixed points. If we denote by $\operatorname{tr} \rho^{*}(\sigma)$ the trace of the representation of $\sigma$ in the space $V^{*}$, then we have the formula:

$$
\rho^{*}(\sigma)=\sum_{i=1}^{r} \frac{\zeta^{2 \nu_{i}}}{1-\zeta^{v_{i}}} .
$$

2.6. Now we shall apply this lemma to our case. Let us suppose that $\zeta^{k}$ appears $\lambda_{k}$ times in the matrix $\rho(\sigma)$, while in the matrix $\rho^{*}(\sigma), \zeta^{k}$ appears $\mu_{k}$ times, where $k=0, \ldots, n-1$. We may evaluate these multiplicities $\lambda_{k}, \mu_{k}$ by the above lemma. But in order to determine $\mu_{0}$ we may use the following lemma owing to Lewittes $[12,13]$.

2.7. Lemma. Let $G$ be a subgroup of the group of automorphisms of $R$, o a generator of $G, r$ the number of ramified points on $R$ over $R^{\prime}$, and $g^{\prime}$ the genus of $R^{\prime}$. Suppose that $2 g^{\prime}-2+r>0$. Then

$$
\mu_{0}=3(g !-1)+r .
$$

In fact, if we fix a function $f_{0}$ on $R^{\prime}$, any quadratic differential is given by $g\left(d f_{0}\right)^{2}$, where $g$ is an arbitrary meromorphic function on $R^{\prime}$. Then we have only to evaluate the number of $g\left(d f_{0}\right)^{2}$ which are everywhere finite in the local 
parameter on $R$. Then we get easily the lemma by the theorem of RiemannRoch and the formula of Riemann-Hurwitz.

2.8. Let us now consider a Riemann surface $R$ defined by the equation:

$$
y^{n}=\prod_{i=1}^{s}\left(x-a_{i}\right)^{k_{i}}, \quad n+k_{1}+\cdots+k_{s}
$$

where $n$ is prime, $1 \leqq k_{i}<n(i=1, \ldots, s)$, and the $a_{i}$ are distinct. Let $\sigma$ be an automorphism of $R$ such that $\sigma(x, y)=(x, \zeta y), \zeta=e^{2 \pi i / n}$. There are $s+1$ fixed points, i.e., $a_{1}, \ldots, a_{s}$ and the point at infinity. At the finite point $a_{i}$, if we take the least positive $l_{i}$ such that $k_{i} l_{i} \equiv 1(\bmod n)$ and put $k_{i} l_{i}=1+n q_{i}$, then $y^{l_{i}} /\left(x-a_{i}\right)^{q_{i}}$ is a local parameter $t$ which is transformed to $t \zeta^{l_{i}}$ by $\sigma$. Therefore $\nu_{i}$ is equal to $l_{i}$. As for the point at infinity, if we take the least $l$ such that $l \cdot \sum_{i=1}^{s} k_{i}+1 \equiv 0(\bmod n)$ and put $l \cdot \sum k_{i}+1=n q$, then $y^{l} / x^{q}$ is a local parameter $t$ which is transformed to $t \zeta^{l}$ by $\sigma$. Therefore $l$ is equal to $\nu_{s+1}$.

We shall give a list which will be necessary in the following.

\section{$R$}

$\begin{array}{llllllllllllll}\nu_{1} & \nu_{2} & \nu_{3} & \nu_{4} & \nu_{5} & \lambda_{1} & \lambda_{2} & \lambda_{3} & \lambda_{4} & \mu_{0} & \mu_{1} & \mu_{2} & \mu_{3} & \mu_{4}\end{array}$

(1) $y^{5}=x(x-1)(x-a)(x-b)$

(2) $y^{5}=x(x-1)(x-a)(x-b)^{3}$ $\begin{array}{lllll}1 & 1 & 1 & 1 & 1\end{array}$

$\begin{array}{lllll}1 & 1 & 1 & 2 & 4\end{array}$

$\begin{array}{lllll}1 & 1 & 2 & 2 & 3\end{array}$

$\begin{array}{lllllllll}3 & 2 & 1 & 0 & 2 & 1 & 5 & 4 & 3\end{array}$

(3) $y^{5}=x(x-1)(x-a)^{3}(x-b)^{3}$

$\begin{array}{lllllllll}2 & 2 & 1 & 1 & 2 & 2 & 4 & 4 & 3\end{array}$

$\begin{array}{lllllllll}2 & 2 & 1 & 1 & 2 & 3 & 3 & 3 & 4\end{array}$

Remark. In the above table $\mu_{0}=3 g^{\prime}-3+r=2$ is common to all these and the number 2 coincides with the number of parameters.

(2) and (3) are contained in the same $\Omega(n, \rho)$, but are not in the same $\Omega\left(n, \rho, \rho^{*}\right)$.

2.9. We are going to classify compact Riemann surfaces of genus 2 with non trivial automorphisms by investigating thoroughly all the possible $\Omega(n, \rho)$. We shall use the same notations as in 1.8 .

(Case $1: p=0$ ). As shown in $1.8, R$ can be expressed in the form

$$
R: y^{n}=\left(x-a_{1}\right)^{m_{1}} \cdots\left(x-a_{s}\right)^{m_{s}}, n+\sum_{i=1}^{s} m_{i}, 1 \leqq m_{i}<n, 1 \leqq i \leqq s,
$$

and $\sigma$ is a map which maps $x$ to $x, y$ to $\zeta y$, where $\zeta=e^{2 \pi i / n}$.

Since $g=2$, the formula (1.8.4) now gives $4=(n-1)(s-1)$ with a prime $n$. Therefore only following three cases (i), (ii), (iii) may occur.

(i) $n=2, s=5: \Omega(2, \rho)$ is a family of $\langle R, \sigma\rangle$, where $R$ is defined by the equation 


$$
y^{2}=x(x-1)\left(x-a_{1}\right)\left(x-a_{2}\right)\left(x-a_{3}\right)
$$

and $\sigma$ is a map which maps $x$ to $x, y$ to $-y$.

(ii) $n=3, s=3: \Omega(3, \rho)$ is a family of $\langle R, \sigma\rangle$, where $R$ are defined by the equations

$$
\left.\begin{array}{cccccccc}
R & \nu_{1} & \nu_{2} & \nu_{3} & \nu_{4} & \lambda_{1} & \lambda_{2} & \langle R, \sigma\rangle \\
\text { (1) } y^{3}=x(x-1)(x-a)^{2} & 1 & 1 & 2 & 2 & 1 & 1 \\
\text { (2) } y^{3}=x(x-1)^{2}(x-a)^{2} & 1 & 2 & 2 & 1 & 1 & 1
\end{array}\right\} \quad\langle(1), \sigma\rangle
$$

and $\sigma$ is a map which maps $x$ to $x, y$ to $\zeta y$, where $\zeta=e^{2 \pi i / 3}$. We can see easily that a Riemann surface of (1) is conformal to a Riemann surface of (2) in a usual sense. We can see easily that $\Omega(3, \rho)=\phi$ if $\rho=[\zeta, \zeta]$ or $\rho=\left[\zeta^{2}, \zeta^{2}\right]$.

\begin{tabular}{|c|c|c|c|c|c|c|c|c|c|}
\hline & $R$ & $\nu_{1}$ & $\nu_{2}$ & $\nu_{3}$ & $\lambda_{1}$ & $\lambda_{2} \lambda$ & & & $\langle R, \sigma\rangle$ \\
\hline (1) & $y^{5}=x(x-1)$ & 1 & 1 & 2 & 1 & 1 & 0 & & \multirow{2}{*}{$\langle(1), \sigma\rangle$} \\
\hline (2) & $y^{5}=x(x-1)^{3}$ & 1 & 2 & 1 & 1 & 10 & ) & & \\
\hline (3) & $y^{5}=x^{2}(x-1)^{4}$ & 3 & 4 & 4 & 0 & 01 & 1 & & \multirow{2}{*}{$\langle(3), \sigma\rangle$} \\
\hline (4) & $y^{5}=x^{4}(x-1)^{4}$ & 4 & 43 & 3 & 0 & 0 & 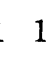 & & \\
\hline (5) & $y^{\overline{5}}=x(x-1)^{2}$ & 1 & 33 & 3 & 1 & 0 & 0 & & \multirow{2}{*}{$\langle(5\rangle, \sigma\rangle$} \\
\hline (6) & $y^{5}=x^{2}(x-1)^{2}$ & 3 & 3 & 1 & 1 & 0 & 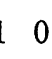 & & \\
\hline (7) & $y^{5}=x^{3}(x-1)^{3}$ & 2 & 2 & 4 & 0 & 1 & & & \multirow{2}{*}{$\langle(7), \sigma\rangle$} \\
\hline (8) & $y^{5}=x^{3}(x-1)^{4}$ & 2 & 2 & 4 & 0 & 14 & & & \\
\hline
\end{tabular}

(iii) $n=5, s=2: \Omega(5, \rho)$ is a family of $\langle R, \sigma\rangle$, where $R$ are defined by the equations

and $\sigma$ is a map which maps $x$ to $x$, and $y$ to $\zeta y$, where $\zeta=e^{2 \pi i / 5}$. We can see easily that all of these Riemann surfaces are conformal to each other from (1) to (4), and all of these are conformal to each other from (5) to (8). However we can show that (1) and (6) are conformal to each other. In fact, if we put

$$
\begin{aligned}
& Y=y^{3} / x(x-1) \\
& X=x
\end{aligned}
$$

then we have

$$
Y^{5}=X(X-1)
$$

And we have also

$$
\begin{aligned}
& y=Y^{2}, \\
& x=X .
\end{aligned}
$$


Here $y^{5}=x^{2}(x-1)^{2}$.

Therefore we conclude that all of these are conformal to each other from (1) to (8) in a usual sense.

(Case $2 ; p=1$ ). Let $r$ be the number of fixed points of $\sigma$. We see by the formula of Hurwitz that $2=(n-1) r$. Only two cases (1) and (2) may occur.

(1) $n=3, r=1:$ By Lemma 2.5 we see easily that this case does not occur.

(2) $n=2, r=2$ : We see that $\rho=[1,-1]$. We shail prove later that $\Omega(2$, $\rho$ ) is not empty as a special case of a general theorem.

2.10. Remark. In the compact Riemann surfaces of genus 2 , we see that any of them cannot have a group of order 84 as a group of automorphisms (cf. $84=84(g-1), g=2)$. Because any of them cannot have an automorphism of order 7 as we see in 2.9, and so we obtain the assertion by a theorem of Sylow. Of course, any compact Riemann surface of genus 2 is found at least in one of these families in 2.9 .

2.11. Remark. We know that any compact Riemann surface of genus $g$ cannot have an automorphism of order more than $10 \mathrm{~g}-10$. Therefore in case of compact Riemann surfaces of genus 3 we have only to study $\Omega(n, \rho)$ for $n=2,3,5,7,11,13,17,19$.

2.12. Remark. $\Omega(n, \rho)=\phi$ if $n=7,11,13,17,19$ and if $\rho=\left[1, \zeta^{\lambda}, \zeta^{\mu}\right] 1 \leqq \lambda$, $\mu \leqq n-1, \zeta=e^{2 \pi i / n}$. In fact, by the lemma 1.2 it is sufficient to prove that $\operatorname{tr} \rho+\operatorname{tr} \bar{\rho}$ is not equal to any rational number. But we see this fact by a simple application of the theory of the Galois group.

2.13. Now we are going to classify compact Riemann surfaces of genus 3 with non-trivial automorphisms in the same way as 2.9.

(Case $1: p=0)$. Since $g=3$, the formula (1.8.4) now gives $6=(n-1)$. $(s-1)$ with a prime $n$, and so only the following three cases (i), (ii), and (iii) may occur.

(i) $n=2, s=7: \Omega(2, \rho)$ is a family of $\langle R, \sigma\rangle$, where $R$ is defined by the equation

$$
y^{2}=x(x-1)\left(x-a_{1}\right)\left(x-a_{2}\right)\left(x-a_{3}\right)\left(x-a_{4}\right)\left(x-a_{5}\right)
$$

and $\sigma$ is a map which maps $x$ to $x, y$ to $-y$. 
(ii) $n=3, s=4: \Omega(3, \rho)$ is a family of $\langle R, \sigma\rangle$, where $R$ are defined by the following equations
$R$ $\begin{array}{lllllllll}\nu_{1} & \nu_{2} & \nu_{3} & \nu_{4} & \nu_{5} & \lambda_{1} & \lambda_{2} & \langle R, \sigma\rangle\end{array}$
(1) $y^{3}=x(x-1)\left(x-a_{1}\right)\left(x-a_{2}\right)$
$\begin{array}{lllll}1 & 1 & 1 & 1 & 2\end{array}$
(2) $y^{3}=x(x-1)\left(x-a_{1}\right)\left(x-a_{2}\right)^{2}$
$\begin{array}{lllll}1 & 1 & 1 & 2 & 1\end{array}$
$\left.\begin{array}{ll}2 & 1 \\ 2 & 1\end{array}\right\}\langle(1), \sigma\rangle$
(3) $y^{3}=x^{2}(x-1)^{2}\left(x-a_{1}\right)^{2}\left(x-a_{2}\right)$
$\begin{array}{lllll}2 & 2 & 2 & 1 & 2\end{array}$
(4) $y^{3}=x^{2}(x-1)^{2}\left(x-a_{1}\right)^{2}\left(x-a_{2}\right)^{2}$
$\begin{array}{lllll}2 & 2 & 2 & 2 & 1\end{array}$
12
$\left.\begin{array}{ll}1 & 2\end{array}\right\}\langle(3), \sigma\rangle$

and $\sigma$ is a map which maps $x$ to $x$ and $y$ to $\zeta y$, where $\zeta=e^{2 \pi i / 3}$. We can see easily that all of these Riemann surfaces are conformal to each other from (1) to (4) in a usual sense.

(iii) $n=7, s=2: \Omega(7, \rho)$ is a family of $\langle R, \sigma\rangle$, where $R$ are defined by the following equations

Group (a) :

$$
R \quad \begin{array}{lllllllllll}
\nu_{1} & \nu_{2} & \nu_{3} & \lambda_{1} & \lambda_{2} & \lambda_{3} & \lambda_{4} & \lambda_{5} & \lambda_{6} & \langle R, \sigma\rangle
\end{array}
$$
(1) $y^{7}=x(x-1)$
$\begin{array}{lll}1 & 1 & 3\end{array}$
(2) $y^{7}=x(x-1)^{5}$
$\begin{array}{lll}1 & 3 & 1\end{array}$
$\left.\begin{array}{llllll}1 & 1 & 1 & 0 & 0 & 0\end{array}\right\}$
(3) $y^{7}=x^{2}(x-1)^{6}$
466
(4) $y^{7}=x^{6}(x-1)^{6}$
$\begin{array}{lll}6 & 6 & 4\end{array}$
$\langle(1), \sigma\rangle$
$\left.\begin{array}{llllll}0 & 0 & 0 & 1 & 1 & 1 \\ 0 & 0 & 0 & 1 & 1 & 1\end{array}\right\}$
$\langle(3), \sigma\rangle$

Group (b) :
(5) $y^{7}=x^{2}(x-1)^{2}$
445
(6) $y^{7}=x^{2}(x-1)^{3}$
454
$\left.\begin{array}{llllll}1 & 0 & 0 & 1 & 1 & 0 \\ 1 & 0 & 0 & 1 & 1 & 0\end{array}\right\}$
$\langle(5), \sigma\rangle$
(7) $y^{7}=x^{4}(x-1)^{3}$
$\begin{array}{lll}2 & 3 & 3\end{array}$
(8) $y^{7}=x^{5}(x-1)^{5}$
$\begin{array}{lll}3 & 3 & 2\end{array}$
$\left.\begin{array}{llllll}0 & 1 & 1 & 0 & 0 & 1 \\ 0 & 1 & 1 & 0 & 0 & 1\end{array}\right\}$
$\langle(7), \sigma\rangle$

Group (c) :
(9) $y^{7}=x^{3}(x-1)^{3}$
(10) $y^{7}=x(x-1)^{3}$
$\begin{array}{lll}5 & 5 & 1\end{array}$
$\left.\begin{array}{llllll}1 & 0 & 1 & 0 & 1 & 0 \\ 1 & 0 & 1 & 0 & 1 & 0\end{array}\right\}$
$\langle(9), \sigma\rangle$
(11) $y^{7}=x^{4}(x-1)^{4}$
155
226
$\begin{array}{llllll}0 & 1 & 0 & 1 & 0 & 1\end{array}$
(12) $y^{7}=x^{4}(x-1)^{6}$
262
$\langle(11), \sigma\rangle$
$\left.\begin{array}{llllll}0 & 1 & 0 & 1 & 0 & 1\end{array}\right\}$

Group (d) :
(13) $y^{7}=x(x-1)^{2}$
(14) $y^{7}=x^{2}(x-1)^{4}$
(15) $y^{7}=x(x-1)^{4}$
(16) $y^{7}=x^{3}(x-1)^{5}$
(17) $y^{7}=x^{5}(x-1)^{6}$
(18) $y^{7}=x^{3}(x-1)^{6}$

142

$\begin{array}{lll}4 & 2 & 1\end{array}$

124

$\begin{array}{lll}5 & 3 & 6\end{array}$

$\left.\begin{array}{llllll}1 & 1 & 0 & 1 & 0 & 0 \\ 1 & 1 & 0 & 1 & 0 & 0 \\ 1 & 1 & 0 & 1 & 0 & 0\end{array}\right\}$

$\langle(13), \sigma\rangle$

365

$\begin{array}{lll}5 & 6 & 3\end{array}$

$\begin{array}{llllll}0 & 0 & 1 & 0 & 1 & 1\end{array}$

$\left.\begin{array}{lllll}0 & 1 & 0 & 1 & 1\end{array}\right\}$

$\langle(16), \sigma\rangle$ 
We can see easily that any two curves of the same group are conformal to each other. Furthermore we can prove that (1) is conformal to (5). In fact, if we put

$$
Y=y^{4} / x(x-1), X=x
$$

then we have

$$
Y^{7}=X(X-1)
$$

And we have also

$$
y=Y^{2}, x=X .
$$

Here $y^{7}=x^{2}(x-1)^{2}$. Furthermore we can prove that (8) is conformal to (9). For the proof we have only to put

$$
Y=y^{4} / x(x-1), X=x .
$$

Here $y^{7}=x^{3}(x-1)^{3}$.

Thus we can conclude that all of these are conformal to each other from (1) to (12) in a usual sense.

(Case $2: p=1$ ). Let $r$ be the number of fixed points of $\sigma$. We see that $6-2=(n-1) r$ by the formula of Riemann-Hurwitz. Therefore only the following three cases may occur.

(1) $n=5, r=1$ : By Lemma 2.5 we see easily that this case does not occur.

(2) $n=3, r=2$ : By Lemma 2.5 we see that the possible $\rho$ is as follows:

$$
\rho_{A}=\left(\begin{array}{lll}
1 & & 0 \\
& \zeta & \\
0 & & \zeta^{2}
\end{array}\right) .
$$

(3) $n=2, r=4:$ By the same Lemma as above, we see that the possible $\rho$ is as follows :

$$
\rho_{B}=\left(\begin{array}{rrr}
1 & & 0 \\
& -1 & -1
\end{array}\right) .
$$

The general equation of $R$ is given in 1.8 (Case $2: p=1$ ). We shall prove later that both $\Omega\left(3, \rho_{A}\right)$ and $\Omega\left(2, \rho_{B}\right)$ are not empty (cf. Theorem 3.2).

(Case $3: p=2$ ). By proposition 2.2 (2) the possible $\rho$ is as follows:

$$
\rho_{C}=\left(\begin{array}{rrr}
1 & & 0 \\
& 1 & \\
0 & & -1
\end{array}\right) .
$$


The general equation of $R$ is given in 1.8 (Case $3: p=2$ ). We shall prove later that $Q\left(2, \rho_{C}\right)$ is not empty.

2.14. Remark. In (Case 2) (1) of 2.13 , we have only to check following $\rho: \rho_{1}=\left[1, \zeta, \zeta^{2}\right], \rho_{2}=\left[1, \zeta, \zeta^{3}\right], \rho_{3}=\left[1, \zeta^{2}, \zeta^{4}\right]$, and $\rho_{4}=\left[1, \zeta^{3}, \zeta^{4}\right]$ by Lemma 1.2.

2.15. Remark. In compact Riemann surfaces of genus 3 , we see that only two types: $y^{7}=x(x-1), y^{7}=x(x-1)^{2}$ have an automorphism of order 7 . Moreover we see that the former cannot attain the maximum of automorphisms of genus 3. Since it is hyperelliptic, we get the assertion by a theorem of R. Tuji [20].

2.16. Remark. It is known by Klein that the Riemann surface defined by the equation: $x y^{3}+y+x^{3}=0$ has the group of automorphisms of order 168 $(=84(p-1) ; p=3)$. Now we can see the Riemann surface defined by the equation $y^{7}=x(x-1)^{2}$ is birationally equivalent to the Riemann surface of Klein. In fact, if we put

$$
x=Y / X, y=Y^{2} / X
$$

then we have

$$
Y^{5}+X^{3}+X Y=0
$$

If we rewrite this equation by $y^{5}+x^{3}+x y=0$, and put

$$
x=Y / X, y=Y^{2} / X
$$

then we have

$$
Y^{7}+X^{2}+X^{3}=0
$$

and so we see that this equation is birationally equivalent to $y^{7}=x(x-1)^{2}$.

2.17. Theorem. There exists one and only one Riemann surface up to conformal equivalence which has a group of automorphisms of order 168 among compact Riemann surfaces of genus 3.

This theorem follows easily from Remark 2.15 and Remark 2.16.

2.18. It would be interesting to study automorphisms of compact Riemann surfaces of higher genera from the view-point of us.

In our study the decision of the surfaces for the cases of $p \geqq 1$ is left to future, since it is beside the main purpose of this paper. 
§ 3. A family of Riemann surfaces: $\Omega\left(g^{\prime}, n,\left\{\nu_{1}, \ldots, \nu_{r}\right\}\right)$

3.1. In view of the results of $\S 2$, it is natural to define certain subfamilies of $\Omega(n, \rho)$ with respect to the exponents $\left\{\nu_{1}, \ldots, \nu_{r}\right\}$. Let $n$ be as before a prime number, and $\left\{\nu_{1}, \ldots, \nu_{r}\right\}$ be a set of positive integers such that $1 \leqq \nu_{i}<n$. Let $\zeta=e^{2 \pi i / n}$. We denote by $\Omega\left(g^{\prime}, n,\left\{\nu_{1}, \ldots, \nu_{r}\right\}\right)$ the set of all isomorphism classes of $(R, \sigma)$ satisfying the following conditions.

(1) $\sigma$ is an automorphism of $R$ of order $n$ with $r$ fixed points.

(2) $R / G$ is of genus $g^{\prime}$, where $G$ is the cyclic group generated by $\sigma$.

(3) Let $t_{i}$ be a local parameter at a point $P_{i}$ of $R$ which is fixed by $\sigma$.

Then $\sigma$ can be represented as $t_{i} \rightarrow \zeta^{\nu} t_{i}+\zeta^{\prime} t_{i}^{2}+\cdots$. Here the coefficient $\zeta^{\nu_{i}}$ is an $n$-th root of unity and does not depend on the choice of local parameters. In this case we shall say that $\sigma$ has the exponents $\left\{\nu_{1}, \ldots, \nu_{r}\right\}$.

3.2. A necessary and sufficient condition for $\Omega\left(g^{\prime}, n,\left\{\nu_{1}, \ldots, \nu_{r}\right\}\right)$ not to be empty. Let $S$ be a compact Riemann surface of genus $g^{\prime}$ and let $(R, \sigma)$ be a cyclic covering of prime order $n$. Let the ramification points on $R$ be $P_{1}, \ldots, P_{r}$ and the projections of them to $S$ be $Q_{1}, \ldots, Q_{r}$ respectively. Let $t_{i}$ be a suitable local parameter at $P_{i}$, then we may assume that

$$
\sigma\left(t_{i}\right)=\zeta^{\nu_{i}} t_{i}, \zeta=e^{2 \pi i / n}
$$

and let $K_{R}$ and $K_{S}$ be the algebraic function fields of $R$ and $S$ respectively. Then there exists an element $y$ of $K_{R}$ such that $\sigma(y)=\zeta y, K_{R}=K_{s}(y)$ and $y^{n} \in K_{s}$.

Let the expansion of $y$ at the point $P_{i}$ be

$$
y=c_{i} t_{i}^{k_{i}}+\cdots,
$$

then we have $\zeta=\zeta^{\nu_{i} k_{i}}$. Therefore we have $\nu_{i} k_{i} \equiv 1(\bmod n)$. On the other hand if we consider the divisor of $y^{n}$ on $S$ we have

$$
\sum_{i=1}^{r} k_{i} Q_{i}+n D
$$

as the divisor. Here $D$ is a divisor on $S$. Now the degree of $\sum_{i=1}^{r} k_{i} Q_{i}+n D$ is

$$
\sum_{i=1}^{n} k_{i}+n \operatorname{deg}(D)=0 .
$$

Therefore we have $\sum_{i=1}^{r} k_{i} \equiv 0(\bmod n)$. This is a necessary condition.

Conversely we shall show that this condition is sufficient. By the assump- 
tion we can put $\sum_{i=1}^{r} k_{i}=n l$, where $l$ is an integer. The degree of the divisor $\sum_{i=1}^{r} k_{i} Q_{i}-n i Q_{0}$ is equal to zero. Here $Q_{0}$ is an arbitrary point on $S$. Now the divisor class group of degree 0 of the algebraic function field $K_{s}$ is isomorphic to a jacobian variety. Since the jacobian variety is a complex torus, there exists the point of order $n$ for an arbitrary point on the variety. Therefore we have an element $z$ of $K_{S}$ and a divisor $E$ of $S$ such that

$$
\sum_{i=1}^{r} k_{i} Q_{i}-n l Q_{0}=n E+(z) .
$$

Then $K_{s}(\sqrt[n]{z})$ is an algebraic function field of our desired Riemann surface.

3.3. Remark. We are now in a position to prove the existence of Riemann surfaces which are left in 2.9 and 2.14.

The non-empty of $\Omega(2, \rho)$ for $\rho=[1,-1]$ is proved as follows. From $n=2$, $r=2$ and $\rho=[1,-1]$ we can derive that $\nu_{1}=1$ and $\nu_{2}=1$. Therefore by the condition $\nu_{i} k_{i} \equiv 1(\bmod 2)$ we see that $k_{1}+k_{2} \equiv 0(\bmod 2)$.

As for the non-empty of $\Omega(2, \rho)$ for $\rho=[1,-1,-1]$ we can derive $\nu_{1}=1$, $\nu_{2}=1, \nu_{3}=1$, and $\nu_{4}=1$ from $n=2, r=4$ and $\rho=[1,-1,-1]$. Therefore we see that $\sum_{i=1}^{4} k_{i} \equiv 0(\bmod 2)$.

The existence of $\Omega(3, \rho)$ for $\rho=\left[1, \zeta, \zeta^{2}\right]$ is shown as follows. We see that $\nu_{1}=1$, and $\nu_{2}=2$. Therefore $k_{1}=1+3 l_{1}$, and $2 k_{2}=1+3 l_{2}$ where $l_{2}$ is an odd number. Then we see that $k_{1}+k_{2}=3\left(1+l_{2}\right) / 2 \equiv 0(\bmod 3)$.

As for the existence of $\Omega(2, \rho)$ for $\rho=[1,1,-1]$ we must recall that $r=0$. Therefore the condition $\sum_{i=1}^{r} k_{i} \equiv 0(\bmod 2)$ is always satisfied.

3.4. $\Omega\left(0, n,\left\{\nu_{1}, \ldots, \nu_{r}\right\}\right)$ is a family of $\langle R, \sigma\rangle$, where $R$ is defined by the equation:

$$
y^{n}=\left(x-a_{1}\right)^{m_{1}} \cdots\left(x-a_{s}\right)^{m_{s}}, n+m_{1}+\cdots+m_{s}, \quad r=s+1,
$$

and $\sigma$ is an automorphism of $R$ such that $(x, y) \rightarrow(x, \zeta y), \zeta=e^{2 \pi i / n}$. Here $m_{i}(i=1, \ldots, s)$ are the numbers which are completely determined by $\left\{\nu_{1}, \ldots, \nu_{r}\right\}$.

Proof. By 1.8 , we see that $R$ is defined by

$$
y^{n}=\left(x-a_{1}\right)^{k_{1}} \cdots\left(x-a_{s}\right)^{k_{s}}, n+k_{1}+\cdots+k_{s} ; 1 \leqq k_{i}<n, i=1, \ldots, s
$$

and $\sigma$ is defined by $(x, y) \rightarrow(x, \zeta y), \zeta=e^{2 \pi i / n}$. Then as is seen in 2.8 , the $k_{i}$ 
are determined by $k_{i} \nu_{i} \equiv 1(\bmod n)$ for $i=1, \ldots, s$ and $\nu_{r}\left(\sum_{i=1}^{s} k_{i}\right)+1 \equiv 0(\bmod$ $n)$.

3.5. Remark. $\Omega\left(g^{\prime}, n,\left\{\nu_{1}, \ldots, \nu_{r}\right\}\right)$ is a subfamily of $\Omega(n, \rho)$, where $\rho$ is a matrix of size $g$ which is determined by the trace formula of Eichler:

$$
\operatorname{tr}(\rho)=1+\sum_{i=1}^{r} \frac{\zeta^{\nu_{i}}}{1-\zeta^{\nu_{i}}}, \text { where } \zeta=e^{2 \pi i / n}
$$

and $g$ is determined by the formula of Riemann-Hurwitz:

$$
2 g-2=n\left(2 g^{\prime}-2\right)+(n-1) r .
$$

3.6. Now we notice here that the number of parameters roughly spoken is equal to

$$
s-2=3 g^{\prime}-3+r .
$$

But this number is equal to $\mu_{0}$, i.e., the number of linearly independent quadratic $G$-invariant differentials (See Lemma 2.7). Therefore it will be natural to construct the theory of Teichmüller space for $\Omega\left(g^{\prime}, n,\left\{\nu_{i}\right\}\right)$.

3.7. For that purpose we begin our study by recalling the definition of Teichmüller space [1]. Let $R_{0}$ be a compact Riemann surface of genus $g \geqq 2$. We consider all pairs $(R, \alpha)$ consisting of compact Riemann surfaces $R$ of genus $g$ and homotopy classes $\alpha$ of topological mappings of $R_{0}$ onto $R$. Two such pairs $(R, \alpha)$ and $\left(R^{\prime}, \alpha^{\prime}\right)$ will be called equivalent if there exists a conformal mapping of $R$ onto $R^{\prime}$ which belongs to the homotopy class $\alpha^{\prime} \alpha^{-1}$. The equivalence class which contains $(R, \alpha)$ will be denoted by $\langle R, \alpha\rangle$. The set of all classes $\langle R, \alpha\rangle$ will be denoted by $T_{g}$, and is called Teichmüller space of genus $g$.

There is another definition of Teichmüller space $T_{g}$. Let us choose a point $p \in R$ and let $\pi_{1}(R, p)$ be the fundamental group formed by the homotopy classes of closed curves from $p$. The group $\pi_{1}(R, p)$ can be generated by $2 g$ generators $A_{1}, B_{1}, \ldots, A_{g}, B_{g}$ which satisfy the single relation

$$
\prod_{i=1}^{g} A_{i} B_{i} A_{i}^{-1} B_{i}^{-1}=1 \text {. }
$$

Any such ordered system of generators is called a canonical system. If $q$ is another point on $R$ we can associate a unique isomorphism of $\pi_{1}(R, p)$ to $\pi_{1}(R, q)$ with every arc $r$ from $p$ to $q$, which is denoted by $T^{r}$. 
Let $\Sigma_{j}$ and $\Sigma_{q}$ be canonical systems for $\pi_{1}(R, p)$ and $\pi_{1}(R, q)$ respectively. We shall say that $\Sigma_{q}$ is equivalent to $\Sigma_{p}$ if and only if $\Sigma_{q}=T^{-} \Sigma_{! \prime}$ for some $\gamma$; the notation means that each generator in $\Sigma_{b}$ is transformed by $T^{r}$ into the corresponding generator in $\Sigma_{q}$.

Suppose that $f$ is a topological mapping of $R$ onto another surface $R^{\prime}$. Then any canonical system $\Sigma$ on $R$ is transformed into a canonical system $f(\Sigma)$ on $R^{\prime}$ formed by the images of the generators. We shall say that $f$ maps $(R, \Sigma)$ onto $\left(R^{\prime}, \Sigma^{\prime}\right)$ if and only if $f(\Sigma)$ is quivalent to $\Sigma^{\prime}$. Moreover if $f$ is a conformal mapping we say that $f$ maps $(R, \Sigma)$ conformally onto $\left(R^{\prime}, \Sigma^{\prime}\right)$ and the two pairs are said to be conformally equivalent. The equivalence class which contains $(R, \Sigma)$ will be denoted by $\langle R, \Sigma\rangle$.

The set of all classes $\langle R, \Sigma\rangle$ defines also a Teichmüller space.

We remark that our two definitions are equivalent. In fact, we know that if $(R, \Sigma)$ and $\left(R^{\prime}, \Sigma^{\prime}\right)$ are two members of the same genus, then there exists a homeomorphism $f: R \rightarrow R^{\prime}$ such that $f$ maps $(R, \Sigma)$ onto $\left(R^{\prime}, \Sigma^{\prime}\right)$ (cf. [4], [14]). And we know that if $f$ maps $(R, \Sigma)$ onto $\left(R^{\prime}, \Sigma^{\prime}\right)$ and $h$ maps $(R, \Sigma)$ onto $\left(R^{\prime}, \Sigma^{\prime}\right)$ then the mappings $f$ and $h$ are homotopic. Conversely, if $h: R \rightarrow R^{\prime}$ is a homeomorphism homotopic to $f$, then $h$ maps $(R, \Sigma)$ onto $\left(R^{\prime}, \Sigma^{\prime}\right)$ (cf. [4]).

Now we denote by $\langle R\rangle$ the class of Riemann surfaces which are conformally equivalent to $R$ and the set of all classes $\langle R\rangle$ will be called the space of moduli and will be denoted by $R_{g}$. Here $g$ means the genus of Riemann surfaces.

3.8. Let $\Omega\left(g^{\prime}, n,\left\{\nu_{1}, \ldots, \nu_{r}\right\}\right)$ the set of all isomorphism classes of $(R, \sigma)$ which is defined in 3.1. Let $(R, \sigma)$ and $\left(R^{\prime}, \sigma^{\prime}\right)$ be two couples such that $\langle R, \sigma\rangle$ and $\left\langle R^{\prime}, \sigma^{\prime}\right\rangle$ belong to $\Omega\left(g^{\prime}, n,\left\{\nu_{i}\right\}\right)$. We say that $(R, \sigma)$ and $\left(R^{\prime}, \sigma^{\prime}\right)$ are topologically equivalent if there exists a topological mapping $f$ of $(R, \sigma)$ onto $\left(R^{\prime}, \sigma^{\prime}\right)$. Here a topological mapping $f$ of $(R, \sigma)$ onto $\left(R^{\prime}, \sigma^{\prime}\right)$ means a topological mapping $f: R \rightarrow R^{\prime}$ such that $f_{\sigma}=\sigma^{\prime} f$. We shall also say that $\langle R, \sigma\rangle$ and $\left\langle R^{\prime}, \sigma^{\prime}\right\rangle$ are topologically equivalent in this case.

3.9. We fix a couple $\left(R_{0}, \sigma_{0}\right)$ such that $\left\langle R_{0}, \sigma_{0}\right\rangle$ belongs to $\Omega\left(g^{\prime}, n,\left\{\nu_{i}\right\}\right)$ and denote by $\Gamma\left(R_{0}, \sigma_{0}\right)$ the set of all the elements $\langle R, \sigma\rangle$ of $\Omega\left(g^{\prime}, n,\left\{\nu_{i}\right\}\right)$ such that $(R, \sigma)$ is topologically equivalent to $\left(R_{0}, \sigma_{0}\right)$.

Let us consider a triple $(R, \sigma, \alpha)$ formed by a couple $(R, \sigma)$ such that $\langle R, \sigma\rangle \in \Gamma\left(R_{0}, \sigma_{0}\right)$, and a homotopy class $\alpha$ of sense preserving topological 
mappings of $\left(R_{0}, \sigma_{0}\right)$ onto $(R, \sigma)$. Here topological mappings $f$ and $g$ of $(R, \sigma)$ into $\left(R^{\prime}, \sigma^{\prime}\right)$ are homotopic means that there exists a continuous mapping $h(z, t)$ of the product $R \times[0,1]$ into $R^{\prime}$ such that $f(z)=h(z, 0), g(z)=h(z, 1)$. It should be noticed that we do not necessarily require the bridge mapping $h$ to satisfy the relation $h \sigma=\sigma^{\prime} h$. Obviously our definition is well defined. We say that $(R, \sigma, \alpha)$ and $\left(R^{\prime}, \sigma^{\prime}, \alpha^{\prime}\right)$ are isomorphic if there exists an isomorphing of $(R, \sigma)$ onto $\left(R^{\prime}, \sigma^{\prime}\right)$ which belongs to the homotopy class $\alpha^{\prime} \alpha^{-1}$. We denote by $\langle R, \sigma, \alpha\rangle$ the isomorphism class of $(R, \sigma, \alpha)$ and the set of all classes $\langle R, \sigma, \alpha\rangle$ is denoted by $\Lambda\left(g^{\prime}, n,\left\{\nu_{i}\right\} ; R_{n}, \sigma_{0}\right)$ or briefly $\Lambda\left(R_{0}, \sigma_{0}\right)$, or $\Lambda$.

The space $I\left(R_{0}, \sigma_{0}\right)$ corresponds to the space of moduli $R_{g}$ and $A\left(R_{0}, \sigma_{0}\right)$ corresponds to the Teichmüller space $T_{\boldsymbol{g}}$.

Now we shall make $\Lambda\left(R_{0}, \sigma_{0}\right)$ into a topological space and furthermore a complex analytic manifold. For that purpose we shall make use of a result of Teichmüller [19].

3. 10. Lemma. Let $\langle R, \alpha\rangle$ and $\left\langle R^{\prime}, \alpha^{\prime}\right\rangle$ be arbitrary two distinct points of $T_{g}$ and choose $(R, \alpha) \in\langle R, \alpha\rangle,\left(R^{\prime}, \alpha^{\prime}\right) \in\left\langle R^{\prime}, \alpha^{\prime}\right\rangle$. Then there exists a quasi conformal mapping $f$ of $R$ onto $R^{\prime}$ which belongs to the homotopy class $\alpha^{\prime} \alpha^{-1}$. Moreover among such mappings there exists one and only one mapping fo for which the minimum of the maximal dilatations is attained.

The dilatation of $f_{0}$ is a constant $K$ and if $f_{0}$ is not conformal, then the characteristic $\mu=\left(f_{0}\right)_{\bar{z}} /\left(f_{0}\right)_{z}$ is given by a regular quadratic differential $\varphi d z^{2}$ on $R$ as

$$
\mu=k \bar{\varphi} /|\varphi|, k=(K-1) /(K+1) \quad(0<k<1) .
$$

Here $\varphi$ is determined uniquely up to a positive factor.

Conversely, if we fix an $(R, \alpha)$ and give a regular quadratic differential $\varphi d z^{2}$ on $R$ and a constant $k(0<k<1)$, then we can determine uniquely an $\left\langle R^{\prime}, \alpha^{\prime}\right\rangle$ and an extremal quasi-conformal mapping $f_{0}:(R, \alpha) \rightarrow\left(R^{\prime}, \alpha^{\prime}\right)$, such that the characteristic $\mu$ of $f_{0}$ is equal to $k \bar{\varphi} /|\varphi|$.

Now we have an analogous proposition to the above in our case.

3.11. Definition. By a quasi-conformal mapping of $(R, \sigma, \alpha)$ to $\left(R_{\cdot}^{\prime}, \sigma^{\prime}, \alpha^{\prime}\right)$, we understand a quasi-conformal mapping $f$ of $R$ to $R^{\prime}$ which belongs to the homotopy class $\alpha^{\prime} \alpha^{-1}$ and such that $f_{\sigma}=\sigma^{\prime} f$. We shall also say that the mapping $f$ is a quasi-conformal mapping of $\langle R, \sigma, \alpha\rangle$ to $\left\langle R^{\prime}, \sigma^{\prime}, \alpha^{\prime}\right\rangle$ in this case. 
3. 12. Proposition. Let $\langle R, \sigma, \alpha\rangle$ and $\left\langle R^{\prime}, \sigma^{\prime}, \alpha^{\prime}\right\rangle$ be topologically equivalent. Then there exists a quasi-conformal mapping of $\langle R, \sigma, \alpha\rangle$ to $\left\langle R^{\prime}, \sigma^{\prime}, \alpha^{\prime}\right\rangle$ and moreover among such mappings there exists one and only one mapping $f_{0}$ for which the minimum of the maximal dilatations is attained.

The dilatation of $f_{0}$ is a constant $K$ and if $f_{0}$ is not conformal then the characteristic $\mu=\left(f_{0}\right)_{\bar{z}} /\left(f_{0}\right)_{z}$ is given by a o-invariant quadratic differential $\varphi d z^{2}$ on $R$ as

$$
\mu=k \bar{\varphi} /|\varphi|, k=(K-1) /(K+1) \quad(0<k<1) .
$$

Here $\varphi$ is determined uniquely up to a positive factor.

Conversely, if we fix $a\langle R, \sigma, \alpha\rangle$ and give a regular quadratic o-invariant differential $\varphi d z^{2}$ on $R$ and a constant $k(0<k<1)$, then we can determine uniquely an $\left\langle R^{\prime}, \sigma^{\prime}, \alpha^{\prime}\right\rangle$ and an extremal quasi-conformal mapping $f_{0}$ such that the characteristic $\mu$ of $f_{0}$ is equal to $k \bar{\varphi} /|\varphi|$.

Proof. We see generally the following fact. Namely, let $\langle R, \sigma, \alpha\rangle$ and $\left\langle R^{\prime}, \sigma^{\prime}, \alpha^{\prime}\right\rangle$ belong to $A\left(R_{0}, \sigma_{0}\right)$ and choose $(R, \sigma) \in\langle R, \sigma\rangle$ and $\left(R^{\prime}, \sigma^{\prime}\right) \in\left\langle R^{\prime}, \sigma\right\rangle$. By virtue of our definition of $A\left(R_{0}, \sigma_{0}\right)$, there exists a topological mapping $f$ of $(R, \sigma)$ onto $\left(R^{\prime}, \sigma^{\prime}\right)$ belonging to the homotopy class $\alpha^{\prime} \alpha^{-1}$, then for all topological mappings $h$ which map $(R, \alpha)$ onto $\left(R^{\prime}, \alpha^{\prime}\right)$ we must have

$$
h \sigma \approx \sigma^{\prime} h \text {. }
$$

In fact, we have $h_{\sigma} \approx f_{\sigma}, f_{\sigma} \approx \sigma^{\prime} f$ and $\sigma^{\prime} f \approx \sigma^{\prime} h$. Therefore we get the assertion. Here the notation $f \approx g$ means that $f$ is homotopic to $g$.

Now let $f_{0}$ be the extremal quasi-conformal mapping of $(R, \alpha)$ onto $\left(R^{\prime}, \alpha^{\prime}\right)$. The existence of $f_{0}$ is guaranteed by Lemma 3.10. Since $f_{0}$ is one of the $h$, which we have considered in the above, so we have

$$
f_{0} \sigma \approx \sigma^{\prime} f_{0} \text {. }
$$

Therefore we have

$$
\sigma^{\prime-1} f_{0} \sigma \approx f_{0}
$$

On the other hand the maximal dilatation of $\sigma^{\prime-1} f_{0} \sigma$ is equal to the maximal dilatation of $f_{0}$. Therefore by the uniqueness of the extremal quasi-conformal mapping we must have

$$
\sigma^{\prime-1} f_{0} \sigma=f_{0} \text { or } f_{0} \sigma=\sigma^{\prime} f_{0}
$$


Therefore we have

$$
\mu(\sigma(z)) \frac{\overline{(d \sigma(z))}}{d \sigma(z)}=\mu(z) \frac{\overline{(d z)}}{d z}
$$

for the corresponding characteristic $\mu$. From these facts we conclude that there exists an extremal quasi-conformal mapping $f_{0}$ and that the corresponding quadratic differential is $\sigma$-invariant.

Now we prove the converse. By Lemma 3.10, under our assumptions we can determine uniquely an $\left\langle R^{\prime}, \alpha^{\prime}\right\rangle$ and an extremal quasi-conformal mapping $f_{0}:(R, \alpha) \rightarrow\left(R^{\prime}, \alpha^{\prime}\right)$ such that the characteristic $\mu$ of $f_{0}$ is equal to $k \bar{\varphi} /|\varphi|$. Then we see that $\mu$ is $\sigma$-invariant and so $f_{0} \sigma f_{0}^{-1}$ is conformal. In fact, we can see easily that if $\mu$ is $\sigma$-invariant, then the characteristic of $f_{0} \sigma$ is $\mu$ and so $f_{0} \sigma f_{0}^{-1}$ is conformal. Therefore if we denote $f_{0} \sigma f_{0}^{-1}$ by $\sigma^{\prime}$, then $\sigma^{\prime}$ is an automorphism of $R^{\prime}$ such that $\sigma^{\prime \prime}=1$. Moreover $\left(R^{\prime}, \sigma^{\prime}, \alpha^{\prime}\right)$ can be considered as a representative of an element of $\Lambda\left(R_{n}, \sigma_{0}\right)$. Because the genus of $R^{\prime}$ is equal to $g$ and the number of fixed points is equal to the number of fixed points of $\sigma$. Since $n$ is prime, it is easy to see that the genus of $R^{\prime} /\left\{\sigma^{\prime}\right\}$ is equal to $g^{\prime}$. Moreover we see that $\nu_{i}=\nu_{i}^{\prime}, i=1, \ldots, r$. In fact, $f_{0}$ is an orientation preserving topological mapping such that $\sigma^{\prime} f_{0}=f_{0} \sigma$ with conformal mappings $\sigma, \sigma^{\prime}$.

We denote the above $\left\langle R^{\prime}, \sigma^{\prime}, \alpha^{\prime}\right\rangle$ by $\langle R, \sigma, \alpha\rangle^{\mu}$ following Bers's notation in the classical theory.

3.13. Teichmüller defines a distance between $\langle R, \alpha\rangle$ and $\left\langle R^{\prime}, \alpha^{\prime}\right\rangle$ by $\log$ $K$, where $K$ is the constant dilatation of the extremal quasi-conformal mapping of $R$ to $R^{\prime}$ in the homotopy class $\alpha^{\prime} \alpha^{-1}$. Now we can also define a similar distance between two arbitrary points $\langle R, \sigma, \alpha\rangle,\left\langle R^{\prime}, \sigma^{\prime}, \alpha^{\prime}\right\rangle$ of $\Lambda\left(R_{0}, \sigma_{0}\right)$ by the same method of Teichmüller. Indeed, we see that there exists one and only one quasi-conformal mapping $f$ of $\langle R, \sigma, \alpha\rangle$ onto $\left\langle R^{\prime}, \sigma^{\prime}, \alpha^{\prime}\right\rangle$ for which the minimum $K$ of the maximal dilatations is attained. Therefore if we put

$$
\operatorname{dis}\left(\langle R, \sigma, \alpha\rangle,\left\langle R^{\prime}, \sigma^{\prime}, \alpha^{\prime}\right\rangle\right)=\log K
$$

then $A\left(R_{0}, \sigma_{0}\right)$ becomes a metric space.

3.14. Theorem. The metric space $\Lambda\left(R_{0}, \sigma_{0}\right)$ is homeomorphic to an $s=3 g^{\prime}-3+r$ dimensional ball $B$.

Proof. Let $\varphi_{1}, \ldots \varphi_{s}$ be a basis of the vector space of $\sigma$-invariant regular quadratic differentials on $R$. Let $\zeta=\left(\zeta_{1}, \ldots, \zeta_{s}\right)$ be an arbitrary point of $B$. 
If we put $|\zeta|=\left(\sum_{j=1}^{*}\left|\zeta_{j}\right|^{2}\right)^{1 / 2}$, and consider a mapping:

$$
B \ni \zeta \rightarrow\langle R, \sigma, \alpha\rangle^{\mu},
$$

where $\mu$ is equal to $|\zeta|\left(\sum_{j=1}^{s} \bar{\zeta}_{j} \bar{\varphi}_{j}\right) /\left|\sum_{j=1}^{s} \zeta_{j} \varphi_{j}\right|$. Then we are able to see that this mapping is topological. In fact, by the classical method we see that it is continuous and by Proposition 3.12, we see that it is a one to one and onto mapping. And as $\zeta$ tends to a boundary point of $B,\langle R, \sigma, \alpha\rangle^{\mu}$ does not tend to any interior points. Therefore our mapping is topological and the assertion is proved.

3.15. We observe that the correspondence $\langle R, \sigma, \alpha\rangle \rightarrow\langle R, \alpha\rangle$ defines a mapping of $\Lambda$ into $T_{g}$, which we denote by . This correspondence is welldefined, that is, if $(R, \sigma, \alpha)$ is isomorphic to $\left(R^{\prime}, \sigma^{\prime}, \alpha^{\prime}\right)$ then obviously $(R, \alpha)$ is equivalent to $\left(R^{\prime}, \alpha^{\prime}\right)$. If $\langle R, \alpha\rangle$ and $\left\langle R^{\prime}, \alpha^{\prime}\right\rangle$ are corresponding points to $\langle R, \sigma, \alpha\rangle$ and $\left\langle R^{\prime}, \sigma^{\prime}, \alpha^{\prime}\right\rangle$ respectively, then we have $h^{\prime} \approx \sigma^{\prime} h$ for every topological mapping $h:(R, \alpha) \rightarrow\left(R^{\prime}, \alpha^{\prime}\right)$. Conversely let $\langle R, \alpha\rangle$ and $\left\langle R^{\prime}, \alpha^{\prime}\right\rangle$ belong to $T_{g}$ and choose $(R, \alpha) \in\langle R, \alpha\rangle$ and $\left(R^{\prime}, \alpha^{\prime}\right) \in\left\langle R^{\prime}, \alpha^{\prime}\right\rangle$. Let $\sigma$ and $\sigma^{\prime}$ be automorphisms of $R$ and $R^{\prime}$ respectively and we assume that there exists a topological mapping $f$ of $(R, \alpha)$ onto $\left(R^{\prime}, \alpha^{\prime}\right)$ such that $f_{\sigma} \approx \sigma^{\prime} f$. Furthermore we assume that $\langle R, \sigma, \alpha\rangle$ is an element of $\Lambda\left(R_{0}, \sigma_{0}\right)$. By 3.12 we see that under our assumption the extremal quasi-conformal mapping $f_{0}$ of $\langle R, \alpha\rangle$ onto $\left\langle R^{\prime}, \alpha^{\prime}\right\rangle$ is a mapping of $(R, \sigma)$ onto $\left(R^{\prime}, \sigma^{\prime}\right)$. Therefore we can conclude that $\left\langle R^{\prime}, \sigma^{\prime}, \alpha^{\prime}\right\rangle$ belongs to $\Lambda\left(R_{0}, \sigma_{0}\right)$ by 3.12 .

3.16. Let $\left\langle R_{0}, \alpha_{0}\right\rangle$ be an element of $T_{g}$ and assume that $\sigma_{0}$ is an automorphism of $R_{0}$ such that $\left\langle R_{0}, \sigma_{0}\right\rangle$ belongs to a family $\Omega\left(g^{\prime}, n,\left\{\nu_{i}\right\}\right)$. Then we denote by $T\left(R_{0}, \sigma_{0}\right)$ all the elements $\langle R, \alpha\rangle$ of $T_{g}$ which satisfy the relation:

$$
f \sigma_{0} \approx \sigma f
$$

with $\sigma$ and $f$ such that $\sigma$ is an automorphism of $R$ and $f$ is a topological mapping of $\left(R_{0}, \alpha_{0}\right)$ onto $(R, \alpha)$.

Now let $T\left(R_{0}, \sigma_{0}\right)$ be naturally topologized.

3.17. The mapping, in 3.15 of $\Lambda\left(R_{0}, \sigma_{0}\right)$ to $T\left(R_{0}, \sigma_{0}\right)$ is bijection and moreover topological. In fact, let $\langle R, \alpha\rangle$ be an arbitrary element of $T\left(R_{0}, \sigma_{0}\right)$. Then $\langle R, \alpha\rangle$ has an automorphism $\sigma$ such that $f_{0} \sigma_{0}=\sigma f_{0}$, where $f_{0}$ is the extremal 
mapping of $\left\langle R_{0}, \alpha_{0}\right\rangle$ to $\langle R, \alpha\rangle$. That is, we can correspond uniquely an element $\langle R, \sigma, \alpha\rangle$ of $A\left(R_{0}, \sigma_{0}\right)$ to $\langle R, \alpha\rangle$. Therefore, is a one-to-one and moreover onto mapping. We see easily that it is topological.

We shall summerize above properties in the following proposition.

3.18. Proposition. Let $\langle R, \alpha\rangle$ be an element of $T\left(R_{0}, \sigma_{0}\right) \subset T_{g}$. If the extremal quasi-conformal mapping $f$ of $\langle R, \alpha\rangle$ onto $\left\langle R^{\prime}, \alpha^{\prime}\right\rangle \in T_{g}$ corresponds to a $\sigma$-invariant regular quadratic differential of $R$, then $\left\langle R^{\prime}, \alpha^{\prime}\right\rangle$ belongs to $T\left(R_{0}, \sigma_{0}\right)$. Conversely, if $\left\langle R^{\prime}, \alpha^{\prime}\right\rangle$ belongs to $T\left(R_{0}, \sigma_{0}\right)$ then the extremal mapping $f$ of $\langle R, \alpha\rangle$ onto $\left\langle R, \alpha^{\prime}\right\rangle$ corresponds to a $\sigma$-invariant regular quadratic differential of $R$.

Proof. The assertion follows easily from 3.12, 3.15, and 3.16.

3.19. Theorem. $T\left(R_{0}, \sigma_{0}\right)$ is an $s\left(=3 g^{\prime}-3+r\right)$ dimensional non-singular analytic submanifold of $T_{\boldsymbol{g}}$.

Proof. Let $\langle R, \alpha\rangle$ be an element of $T\left(R_{0}, \sigma_{0}\right)$ and let $\varphi_{j} d z^{2}(j=1, \ldots, 3 g-3)$ be a basis of regular quadratic differentials of $R$. We may sssume that the first $3 g^{\prime}-3+r$ of the above basis are $\sigma$-invariant. Now let $\rho|d z|(\rho>0)$ be a $\sigma$-invariant metric, then by a theorem of Weil [21] for sufficiently small $c \mu_{j}=c \bar{\varphi}_{j} / \rho$ $\left(j=1, \ldots, 3 g^{\prime}-3\right)$ is a Beltrami basis of $R$. The first $3 g^{\prime}-3+r$ of the above Beltrami basis are $\sigma$-invariant.

It is known by Bers [3] that for $\mu=\sum_{j=1}^{3 g-3} z_{j} \mu_{j}$

$$
\left(z_{1}, \ldots, z_{3 g-3}\right) \rightarrow\langle R, \alpha\rangle^{\mu}
$$

is a $C^{\infty}$-homeomorphism of a neighborhood of the origin of the complex number space $C^{3 g-3}$ onto a neighborhood of $\langle R, \alpha\rangle$. We call these $z_{j}$ the coordinates associated with complex Beltrami basis $\mu_{j}(j=1, \ldots, 3 g-3)$. Then it is known that the coordinates associated with Beltrami basis are complex analytic coordinates in $T_{g}$.

Now introduce the complex analytic structure in a neighborhood $U$ of any point $\langle R, \alpha\rangle$. Then by Proposition 3.18 we can show that $U \cap T\left(R_{0}, \sigma_{0}\right)$ is identical with all points $\left(z_{1}, \ldots, z_{s}, 0, \ldots, 0\right)$. Thus we get the assertion.

3.20. Theorem. If $3 g^{\prime}-3+r$ is equal to one, then $T\left(R_{0}, \sigma_{0}\right)$ is holomorphically equivalent to the upper-half plane (or the interior of the unit circle).

Proof. By Theorem 3.19, $T\left(R_{0}, \sigma_{0}\right)$ is a one dimensional manifold and by 
Theorem 3.14 it is simply connected. Therefore it is holomorphically equivalent to either the unit circle or the punctured complex plane.

However it is known that $T_{\boldsymbol{g}}$ is holomorphically equivalent to a bounded domain in complex number space (cf. [5]). Therefore there are non-constant bounded and holomorphic functions on $T\left(R_{0}, \sigma_{0}\right)$ and so we conclude that it is holomorphically equivalent to the upper-half plane by the theorem of Liouville.

3.21. Theorem. $\Lambda\left(R_{0}, \sigma_{0}\right)$ is a $3 g^{\prime}-3+r$ dimensional complex analytic manifold.

Proof. By 3.17 and Theorem 3.19 we can endow $\Lambda\left(R_{0}, \sigma_{0}\right)$ with an analytic structure such that the natural mapping $c$ is holomorphic. In fact, $T\left(R_{0}, \sigma_{0}\right)$ is a non-singular analytic submanifold of $T_{g}$.

\section{$\S 4$. Embedding of $\Lambda$ into a bounded symmetric domain}

4.1. In 3.9 we fix an element $\left\langle R_{0}, \sigma_{0}\right\rangle$ in $\Omega\left(g^{\prime}, n,\left\{\nu_{i}\right\rangle\right)$, and we denote by $\Gamma\left(R_{0}, \sigma_{0}\right)$ all the elements of $\Omega$ which are topologically equivalent to $\left\langle R_{0}, \sigma_{0}\right\rangle$. However if $g^{\prime}=0$, then we have the following lemma.

4.2. Lemma. Any two elements of $\Omega\left(0, n,\left\{\nu_{1}, \ldots, \nu_{r}\right\}\right)$ are topologically equivalent.

Proof. By 3.4, $\Omega\left(0, n,\left\{\nu_{i}\right\}\right)$ is a family of $\langle R, \sigma\rangle$, where $R$ is defined by the equation:

$$
y^{n}=\left(x-a_{1}\right)^{m_{1}}\left(x-a_{2}\right)^{m_{2}} \cdots\left(x-a_{s}\right)^{m_{s}}, n+m_{1}+\cdots+m_{s}, \quad r=s+1,
$$

and $\sigma$ is an automorphism of $R$ such that $(x, y) \rightarrow(x, \zeta y), \zeta=e^{2 \pi i / n}$. Then $m_{i}(i=1, \ldots, r)$ are completely determined by $\nu_{1}, \ldots, \nu_{r}$.

Now taking a suitable branch, we may assume that

$$
\log y=\frac{1}{n} \sum_{k=1}^{s} m_{k} \log \left(x-a_{k}\right) \text {. }
$$

Let $y_{0}\left(q_{0}, x\right), \ldots, y_{n-1}\left(q_{0}, x\right)$ be $n$ function elements at an ordinary point $q_{0} \in R /\{\sigma\}$. We may assume that $\zeta y_{0}=y_{1}, \zeta^{2} y_{0}=y_{2}, \ldots, \zeta^{n-1} y_{0}=y_{n-1}$. Let $K_{v}$ be a curve on $R$ from $p_{0}$ to $\sigma^{\nu} p_{0}$ such that the projection $C_{\nu}$ does not pass through any branch points $a_{1}, \ldots, a_{s}, \infty$. Where $p_{0}$ is a point over $q_{0}$. Performing continuation along $C_{\imath}$ we have 


$$
\begin{aligned}
\int_{C_{\nu}} d \log y_{0}\left(q_{0}, x\right) & =\frac{1}{n} \sum_{k=1}^{s} m_{k} \int_{C_{\nu}} \frac{d x}{x-a_{k}} \\
& =\frac{2 \pi i}{n} \sum_{k=1}^{s} m_{k} \mathrm{I}\left(a_{k}, C_{\nu}\right) .
\end{aligned}
$$

where $I\left(a_{k}, C_{\nu}\right)$ is the index of the curve $C_{\nu}$ with respect to $a_{k}$. By the continuation, $y_{0}(x)$ is transformed to $y_{\nu}(x)$. Then we have

$$
\log y_{\nu}\left(q_{0}, x\right)-\log y_{0}\left(q_{0}, x\right) \equiv \frac{2 \pi i}{n} \sum_{k=1}^{s} m_{k} I\left(a_{k}, C_{\nu}\right) \quad(\bmod 2 \pi i) .
$$

But by the assumption we have $y_{\nu}(x)=\zeta^{\nu} y_{0}(x)$. Therefore we have

$$
\log \zeta^{\nu} \equiv \frac{2 \pi i}{n} \sum_{k=1}^{s} m_{k} I\left(a_{k}, C_{\nu}\right) \quad(\bmod 2 \pi i)
$$

and so we obtain

$$
\nu \equiv \sum_{k=1}^{s} m_{k} I\left(a_{k}, C_{\nu}\right) \quad(\bmod n)
$$

This relation is fundamental in our argument.

Now we construct a topological mapping $f: R \rightarrow R^{\prime}$ which satisfies $f_{\sigma}=\sigma^{\prime} f$, provided that $\langle R, \sigma\rangle$ and $\left\langle R^{\prime}, \sigma^{\prime}\right\rangle$ belong to $\Omega\left(0, n,\left\{\nu_{i}\right\}\right)$. We may assume that $R^{\prime}$ is defined by

$$
y^{\prime n}=\left(x^{\prime}-a_{1}^{\prime}\right)^{m_{1}} \cdots\left(x^{\prime}-a_{s}^{\prime}\right)^{m_{s}}
$$

and $\sigma^{\prime}$ is an automorphism of $R^{\prime}$ such that $\left(x^{\prime}, y^{\prime}\right) \rightarrow\left(x^{\prime}, \zeta y^{\prime}\right), \zeta=e^{2 \pi i / n}$. Let $S$ and $S^{\prime}$ be $R /\{\sigma\}$ and $R^{\prime} /\left\{\sigma^{\prime}\right\}$ respectively. Let $I$ and $\Pi^{\prime}$ be the natural projections of $R$ to $S$ and of $R^{\prime}$ to $S^{\prime}$ respectively.

We know that there is a diffeomorphism $g$ of $S$ to $S^{\prime}$ which takes $a_{i}$ into $a_{i}^{\prime}, i=1, \ldots, s$. Then we have a local diffeomorphism:

$$
\stackrel{-1}{\Pi}^{\prime} g \Pi: p \rightarrow p^{\prime},
$$

such that $p_{0} \rightarrow p_{0}^{\prime}$. Let $\Pi\left(p_{0}\right)$ be $q_{0}$, and $g\left(q_{0}\right)$ be $q_{0}^{\prime}$. Let $K_{1}$ be a curve from $p_{0}$ to a point $p$ such that the projection $C_{1}$ does not pass through any branch points. Let $K_{2}$ be another such curve and $C_{2}$ be the projection. Then we denote by $C$ the projection of the closed curve $K_{2}^{-1} K_{1}$ from $p_{0}$ to $p_{0}$ on $R$. By the above formula $(4.2 .1)$ we have

$$
\sum_{k=1}^{s} m_{k} I\left(a_{k}, C\right) \equiv 0 \quad(\bmod n)
$$


for the closed curve $C$.

Now put $C_{1}^{\prime}=g\left(C_{1}\right), C_{2}^{\prime}=g\left(C_{2}\right)$, and $C^{\prime}=g(C)$. Then we have by the formula $(4.2 .2)$

$$
\sum_{k=1}^{s} m_{k} I\left(a_{k}^{\prime}, C^{\prime}\right) \equiv 0 \quad(\bmod n)
$$

If we lift $C_{1}^{\prime}$ to a curve from $p_{0}^{\prime}$ to $p^{\prime}$ and $C_{2}^{\prime}$ to a curve from $p_{0}^{\prime}$ to $p^{\prime \prime}$ then by (4.2.3) we see that $p^{\prime}$ must coincide with $p^{\prime \prime}$. Therefore we can define a mapping $f$ of $R$ to $R^{\prime}$.

Now let $K$ be a curve on $R$ from $p_{0}$ to $\sigma p_{0}$. The projection of $K$ is a closed curve $C$ from $q_{0}$ to $q_{0}$, and so we obtain by the formula (4.2.1)

$$
1 \equiv \sum_{k=1}^{s} m_{k} I\left(a_{k}, C\right) \quad(\bmod n) .
$$

Therefore we obtain also

$$
1 \equiv \sum_{k=1}^{s} m_{k} I\left(a_{k}^{\prime}, C^{\prime}\right) \quad(\bmod n) .
$$

From this we can assert that $f$ commute with given automorphisms.

4.3. By Lemma 4.2, using the whole members of $\Omega\left(0, n,\left\{\nu_{1}, \ldots, \nu_{r}\right\}\right)$ we are able to construct the generalized Teichmüller space $\Lambda\left(R_{0}, \sigma_{0}\right)$.

Now we may say that one of our main problems is to investigate the holomorphy of parameters $a_{1}, \ldots, a_{s}$ of Riemann surfaces :

$$
y^{n}=\left(x-a_{1}\right)^{m_{1}}\left(x-a_{2}\right)^{m_{2}} \cdots\left(x-a_{s}\right)^{m_{s}}, n+\sum_{k=1}^{s} m_{k}, r=s+1,
$$

with respect to the complex structure of the generalized Teichmüller space $A$.

For that purpose we begin by studying the periods of the integrals of the first kind on $R$.

4.4. Now let $\langle R, \sigma\rangle$ be an element of $\Omega\left(0, n,\left\{\nu_{1}, \ldots, \nu_{r}\right\}\right)$. By Lemma 2.5 , we have a matrix representation of $\sigma$ in the form:

$$
\phi(\zeta)=\left|\begin{array}{lll}
\zeta^{\alpha_{1}} & & 0 \\
& \cdot & \\
& \cdot & \\
0 & & \zeta^{\alpha_{g}}
\end{array}\right| .
$$

We must notice here that $\zeta^{\alpha_{1}}, \ldots, \zeta^{\alpha_{g}}$ are primitive $n$-th roots of unity and 
any of them is not equal to 1 . Now we denote by $\lambda_{0}$ a point $\left\langle R_{0}, \sigma_{0}, \alpha_{0}\right\rangle$ and we shall find a suitable system of basis $\omega_{1}(\lambda), \ldots, \omega_{g}(\lambda)$ of differentials of the first kind such that we get the same representation $\phi(\zeta)$ for all $\lambda$ in a neighborhood $V\left(\lambda_{0}\right)$ with $\omega_{1}(\lambda), \ldots, \omega_{g}(\lambda)$ in the following $4.5-4.8$.

4.5. Lemma (Bers [6]). Let $T_{g}$ be the Teichmüller space. One can associate to every $\tau \in T_{g}$ a bounded Jordan domain $B(\tau)$ and $2 g$ Möbius transformations $z \rightarrow A_{j}(z, \tau), z \rightarrow B_{j}(z, \tau), j=1, \ldots, g$ such that the following conditions are satisfied.

(1) The $A_{j}$ and $B_{j}$ depend holomorphically on $\tau$ and satisfy the relation:

$$
\prod_{j=1}^{g} A_{j} B_{j} A_{j}^{-1} B_{j}^{-1}=1 \text {. }
$$

For every fixed $\tau \in T_{g}$ they generate, with single defining relation (4.5.1), a fixed point free discrete group $G(\tau)$ of conformal self-mappings of $B(\tau)$, so that $R(\tau)=B(\tau) / G(\tau)$ is a compact Riemann surface of genus $g . \quad R\left(\tau_{0}\right)$ is the surface $R$.

(2) Denote by $\Sigma(\tau)$ the basis of the fundamental group of $R(\tau)$ defined by $A_{1}, \ldots, B_{g}$ and by $f_{\tau_{0} \tau}$ a quasi-conformal mapping of $R_{0}$ onto $R(\tau)$ which takes $\Sigma\left(\tau_{0}\right)$ into $\Sigma(\tau)$. Then the point $\tau$ corresponds to the couple $\left\langle R(\tau),\left(f_{\tau_{0} \tau}\right)\right\rangle$, here ( $f$ ) means the homotopy class of $f$.

(3) If we denote by $W(\tau)$ the complex vector space of holomorphic functions $\varphi(z), z \in B(\tau)$ for which $\varphi(z) d z$ is invariant under $G(\tau)$ there exist in $W(\tau), g$ distinguished elements, $p_{k}(z, \tau)$ determined by the conditions

$$
\int_{z}^{A_{i}(z, \tau)} p_{k}\left(z^{\prime}, \tau\right) d z^{\prime}=\delta_{i k}
$$

these correspond to the normalized Abelian differentials of the first kind on $R(\tau)$ belonging to the "canonical" homology basis $\tilde{\Sigma}(\tau)$ determined by $\Sigma(\tau)$. The period matrix of $R(\tau)$ belonging to $\bar{\Sigma}(\tau)$ will be denoted by $\left(\pi_{i j}(\tau)\right)$. It has the elements

$$
\pi_{i j}(\tau)=\int_{z}^{R_{i}(z, \tau)} p_{j}\left(z^{\prime}, \tau\right) d z^{\prime}
$$

and is a point in the Siegel space of symmetric matrices with positive definite imaginary part.

(4) If we denote by $M$ the domain in a complex number space of $3 g-2$ dimensions which consists of points $(z, \tau)$ with $z \in B(\tau)$ and $\tau \in T_{g}$, then the 
functions $p_{k}(z, \tau), k=1, \ldots, g$ are holomorphic in $M$, and the mapping $\tau \rightarrow\left(\pi_{i j}(\tau)\right)$ of the Teichmüller space into the Siegel space is holomorphic.

Now let us apply the above lemma to our case.

4.6. Let $\left(R^{*}, \sigma^{*}\right)$ define an element $\lambda^{*}$ of $\Lambda$, and let $(R, \sigma)$ define an element $\lambda$ of $\Lambda$. As seen in Theorem 3.21 there exists an holomorphic injection $6: \Lambda \rightarrow T_{g}$. Put $\iota\left(\lambda^{*}\right)=\tau^{*}$, and $\iota(\lambda)=\tau$. Then we can take $R\left(\tau^{*}\right)$ for $R^{*}$ and take $R(\tau)$ for $R$. Let $H_{1}(R(\tau), Z)$ be one dimensional homology group of $R(\tau)$ with integral coefficients. Let $\varphi_{*}(\sigma ; \lambda)$ denote the endomorphism of $H_{1}(R(\tau), Q)$ induced naturally by the automorphism $\sigma$ of $R(\tau)$, where $Q$ is the rational number field.

By Lemma $4.5, \tau={ }_{\iota}(\lambda)$ corresponds to $\left\langle R(\tau),\left(f_{\tau^{*} \tau}\right)\right\rangle$ and every member of $\left(f_{\tau^{*} \tau}\right)$ takes $\Sigma\left(\tau^{*}\right)$ into $\Sigma(\tau)$. And the extremal quasi-conformal mapping $f$ of $R\left(\tau^{*}\right)$ onto $R(\tau)$ which belongs to $\left(f_{\tau^{*} \tau}\right)$ takes not only $\Sigma\left(\tau^{*}\right)$ into $\Sigma(\tau)$ but also commute with the automorphisms; that is, we have

$$
f \sigma^{*}=\sigma f
$$

This $f$ induces an isomorphism $f_{*}$ :

$$
H_{1}\left(R\left(\tau^{*}\right), Q\right) \rightarrow H_{1}(R(\tau), Q) .
$$

Furthermore we can see that the following commutative diagram:

$$
\begin{array}{r}
H_{1}\left(R\left(\tau^{*}\right), Q\right) \stackrel{f_{*}}{\longrightarrow} H_{1}(R(\tau), Q) \\
\downarrow \varphi_{*}\left(\sigma^{*}, \lambda^{*}\right) r \varphi_{*}(\sigma, \lambda) \\
H_{1}\left(R\left(\tau^{*}\right), Q\right) \stackrel{f_{*}}{\rightarrow} H_{1}(R(\tau), Q)
\end{array}
$$

hold. In fact, from $f_{\sigma^{*}}=\sigma f$. we can easily obtain $f_{*} \varphi_{*}\left(\sigma^{*} ; \lambda^{*}\right)=\varphi_{*}(\sigma ; \lambda) f_{*}$.

Let $\widetilde{A}_{i}(z, \tau)$ be the homology class which is defined by $A_{i}(z, \tau)$, and $\widetilde{B}_{i}(z, \tau)$ be the homology class which is defined by $B_{i}(z, \tau)$. Now put

$$
\left(\begin{array}{c}
p_{1}(z, \tau)_{\sigma} \\
\cdot \\
\cdot \\
p_{g}(z, \tau)_{\sigma}
\end{array}\right)=\left(\begin{array}{c}
\cdots \\
a_{i j}(\tau) \\
\cdots
\end{array}\right)\left(\begin{array}{c}
p_{1}(z, \tau) \\
p_{g}(z, \tau)
\end{array}\right),
$$

or simply $P(z, \tau)_{\sigma}=A(\tau) P(z, \tau)$, then we have

$$
a_{i j}(\tau)=\int_{\tilde{A}_{j}(z, \tau)}\left(p_{i}(z, \tau) d z\right)_{\sigma}=\int_{\vartheta_{*}(\sigma ; \lambda) \tilde{A}_{j}(z, \tau)} p_{i}(z, \tau) d z
$$


Since $\varphi_{*}(\sigma, \lambda) \tilde{A}_{j}(z, \tau) \in H_{1}(R(\tau), Q)$ we may write

$$
\varphi_{*}(\sigma, \lambda) \widetilde{A}_{j}(z, \tau)=\sum_{i=1}^{q} \alpha_{j i}(\tau) \widetilde{A}_{i}(z, \tau)+\sum_{i=1}^{q} \beta_{j i}(\tau) \widetilde{B}_{i}(z, \tau) .
$$

It is easy to see that $\alpha_{j i}(\tau)$ and $\beta_{j i}(\tau)$ are constants. In fact, we have

$$
\begin{aligned}
\varphi_{*}(\sigma, \lambda) f_{*} \tilde{A}_{j}\left(z, \tau^{*}\right) & =\varphi_{*}(\sigma, \lambda) \widetilde{A}_{j}(z, \tau) \\
& =\sum_{i=1}^{g} \alpha_{j i}(\tau) \widetilde{A}_{i}(z, \tau)+\sum_{i=1}^{g} \beta_{j i}(\tau) \widetilde{B}_{i}(z, \tau)
\end{aligned}
$$

and on the other hand we have

$$
\begin{aligned}
f_{*} \varphi_{*}\left(\sigma^{*}, \lambda^{*}\right) \widetilde{A}_{j}\left(z, \tau^{*}\right) & =f_{*}\left(\sum_{i=1}^{g} \alpha_{j i}\left(\tau^{*}\right) \widetilde{A}_{i}\left(z, \tau^{*}\right)+\sum_{i=1}^{q} \beta_{j i}\left(\tau^{*}\right) \widetilde{B}_{i}\left(z, \tau^{*}\right)\right) \\
& =\sum_{i=1}^{g} \alpha_{j i}\left(\tau^{*}\right) \widetilde{A}_{i}(z, \tau)+\sum_{i=1}^{q} \beta_{j i}\left(\tau^{*}\right) \widetilde{B}_{i}(z, \tau) .
\end{aligned}
$$

Therefore we obtain

$$
\alpha_{j i}(\tau)=\alpha_{j i}\left(\tau^{*}\right) \text { and } \beta_{j i}(\tau)=\beta_{j i}\left(\tau^{*}\right)
$$

Then by Lemma 4.5 , we see that

$$
\begin{aligned}
a_{i j}(\tau) & =\int_{\tilde{A}_{j}(z, \tau)}\left(p_{i}(z, \tau) d z\right) \sigma \\
& =\int_{\Sigma x_{j k} \tilde{A}_{k}(z, \tau)} p_{i}(z, \tau) d z+\int_{\Sigma \beta_{j k} \tilde{R}_{k}(z, \tau)} p_{i}(z, \tau) d z \\
& =\sum_{k} \alpha_{j k}\left(\tau^{*}\right) \delta_{k i}+\sum_{k} \beta_{j k}\left(\tau^{*}\right) \pi_{k i}(\tau)
\end{aligned}
$$

and so all $a_{i j}(\tau)$ are holomorphic in $T\left(R_{0}, \sigma_{0}\right)$. By Theorem 3.21 $\tau=\iota(\lambda)$, is holomorphic. Therefore $A(\iota(\lambda))$ is a holomorphic matrix in $\Lambda\left(R_{0}, \sigma_{0}\right)$. We denote this matrix by $A^{*}(\lambda)$ and the vector $P(z, \ell(\lambda))$ by $P^{*}(z, \lambda)$.

By our assumption we may assume that $A^{*}(\lambda)$ is equivalent to $\phi(\zeta)$ for all $\lambda \in \Lambda\left(R_{0}, \sigma_{0}\right)$. Then we have the following proposition.

4.7. Proposition. Notations being as above, for an arbitrary point $\lambda^{*} \in \Lambda$, there exists a non-singular and holomorphic matrix $X(\lambda)$ in a sufficiently small neighborhood $V\left(\lambda^{*}\right)$ such that

$$
X^{-1}(\lambda) A^{*}(\lambda) X(\lambda)=\phi(\zeta) .
$$

Proof. Let $f(x)$ be the characteristic polynomial of $\phi(\zeta)$, and put

$$
f(x)=\prod_{i=1}^{r}\left(x-\zeta_{i}\right)^{n_{i}},
$$


where the $\zeta_{i}$ runs over all the distinct elements of the diagonal of $\phi(\zeta)$ in 4.4 . If we put

$$
f_{i}(x)=\prod_{j \neq i}\left(x-\zeta_{j}\right)^{n_{j}},
$$

then there are $r$ polynomials $P_{1}(x), \ldots, P_{r}(x)$ such that

$$
P_{1}(x) f_{1}(x)+\cdots+P_{r}(x) f_{r}(x)=1 .
$$

Therefore we have

$$
P_{1}\left(A^{*}(\lambda)\right) f_{1}\left(A^{*}(\lambda)\right)+\cdots+P_{r}\left(A^{*}(\lambda)\right) f_{r}\left(A^{*}(\lambda)\right)=I .
$$

Put $P_{i}\left(A^{*}(\lambda)\right) f_{i}\left(A^{*}(\lambda)\right)=S_{i}\left(A^{*}(\lambda)\right)$. By the simple application of the theory of linear algebra we can choose $g$ vectors $\mathfrak{x}_{1}, \ldots, \mathfrak{x}_{g}$ such that

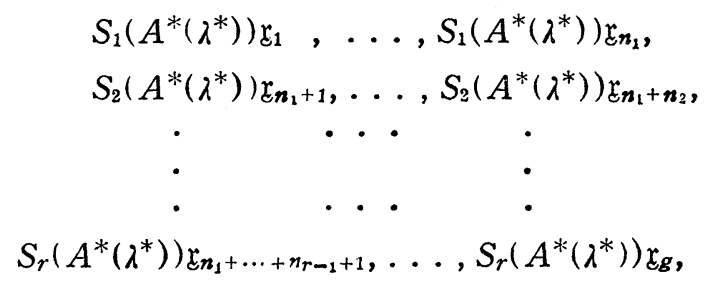

are linearly independent. Now if we put

$$
X(\lambda)=\left(S_{1}\left(A^{*}(\lambda)\right) \mathfrak{x}_{1}, \ldots, S_{r}\left(A^{*}(\lambda)\right) \mathfrak{x}_{g}\right)
$$

then $X(\lambda)$ is not only a holomorphic matrix in $\lambda$ but also a non-singular matrix in a sufficiently small neighborhood $V\left(\lambda^{*}\right)$.

Thus we see that

$$
\begin{aligned}
A^{*}(\lambda) X(\lambda) & =\left(A^{*}(\lambda) S_{1}\left(A^{*}(\lambda)\right) \mathfrak{x}_{1}, \ldots, A^{*}(\lambda) S_{r}\left(A^{*}(\lambda)\right) \mathfrak{x}_{g}\right) \\
& =\left(\zeta^{\alpha_{1}} S_{1}\left(A^{*}(\lambda)\right) \mathfrak{x}_{1}, \ldots, \zeta^{\alpha_{r}} S_{r}\left(A^{*}(\lambda)\right) \mathfrak{x}_{g}\right) \\
& =X(\lambda) \phi(\zeta) .
\end{aligned}
$$

4.8. Now we are in a position to prove the assertion stated in 4.4. Put

$$
X^{-1}(\lambda) P^{*}(z, \lambda)=\omega(z, \lambda)={ }^{t}\left(\omega_{1}(z, \lambda), \ldots, \omega_{g}(z, \lambda)\right),
$$

then for all $\lambda \in V\left(\lambda^{*}\right)$ we have the desired system $\omega(z, \lambda)$. Namely we have

$$
\begin{aligned}
\omega(z, \lambda) \sigma & =\left(X^{-1}(\lambda) P^{*}(z, \lambda)\right)_{\sigma} \\
& =X^{-1}(\lambda) A^{*}(\lambda) P^{*}(z, \lambda) \\
& =\phi(\zeta) X^{-1}(\lambda) P^{*}(z, \lambda),
\end{aligned}
$$


therefore we see that

$$
\omega(z, \lambda)_{\sigma}=\phi(\zeta) \omega(z, \lambda)
$$

for all $\lambda \in V\left(\lambda^{*}\right)$.

4.9. Every element $a$ of $Q(\zeta)$ is of a form: $a=a_{0}+a_{1} \zeta+\cdots+a_{n-2} \zeta^{n-2}$, where $a_{0}, a_{1}, \ldots, a_{n-2} \in Q$. If we define $\varphi_{*}(a ; \lambda)$ by

$$
\varphi_{*}(a ; \lambda)=a_{0}+a_{1} \varphi_{*}(\sigma ; \lambda)+\cdots+a_{n-2} \varphi_{*}(\sigma ; \lambda)^{n-2},
$$

then naturally we have

$$
\varphi_{*}(\zeta ; \lambda)=\varphi_{*}(\sigma ; \lambda) \text {. }
$$

Now $\varphi_{*}\left(a ; \lambda^{*}\right), a \in K=Q(\zeta)$ operates on $H_{1}\left(R\left(\tau^{*}\right), Q\right)$, and so we can find $u=g / h$ vectors $Z_{1}\left(\lambda^{*}\right), \ldots, Z_{u}\left(\lambda^{*}\right)$ such that

$$
H_{1}\left(R\left(\tau^{*}\right), Q\right)=\varphi_{*}\left(K ; \lambda^{*}\right) Z_{1}\left(\lambda^{*}\right) \oplus \cdots \oplus \varphi_{*}\left(K ; \lambda^{*}\right) Z_{u}\left(\lambda^{*}\right),
$$

where $[K: Q]=n-1=2 h$, and $\oplus$ means the direct sum.

If we denote by $Z_{i}(\lambda)$ the images of $Z_{i}\left(\lambda^{*}\right)$ by the mapping $f_{*}$, then we have

$$
H_{1}(R(\tau), Q)=\varphi_{*}(K ; \lambda) Z_{1}(\lambda) \oplus \cdots \oplus \varphi_{*}(K ; \lambda) Z_{u}(\lambda) .
$$

Now if we put

$$
\mathfrak{x}_{1}(\lambda)=\int_{Z_{1}(\lambda)} \omega(z, \lambda), \ldots, \mathfrak{x}_{u}(\lambda)=\int_{z_{u}(\lambda)} \omega(z, \lambda),
$$

then $\mathfrak{x}_{1}(\lambda), \ldots, \mathfrak{x}_{u}(\lambda)$ are holomorphic vectors of $\lambda$ in $V\left(\lambda^{*}\right)$. In fact, we can put

$$
Z_{i}\left(\lambda^{*}\right)=\sum_{k=1}^{g} a_{k}^{i} \widetilde{A}_{k}\left(z, \tau^{*}\right)+\sum_{k=1}^{q} b_{k}^{i} \widetilde{B}_{k}\left(z, \tau^{*}\right) \quad .(1 \leqq i \leqq u)
$$

with rational coefficients. Since the mapping $f_{*}$ is a $Q$-isomorphism, and so we have

$$
Z_{i}(\lambda)=\sum_{k=1}^{g} a_{k}^{i} \widetilde{A}_{k}(z, \tau)+\sum_{k=1} b_{k}^{i} \widetilde{B}_{k}(z, \tau)
$$

Therefore we have

$$
\begin{aligned}
\mathfrak{x}_{i}(\lambda) & =\int_{z_{\boldsymbol{k}}(\lambda)} X^{-1}(\lambda) P\left(z^{\prime}, \tau\right) d z^{\prime} \\
& =X^{-1}(\lambda)\left(\sum_{k=1}^{g} a_{k}^{i} \int_{\tilde{A}_{k}(z, \tau)} P\left(z^{\prime}, \tau\right) d z^{\prime}+\sum_{k=1}^{g} b_{k}^{i} \int_{\tilde{B}_{k}(z, \tau)} P\left(z^{\prime}, \tau\right) d z^{\prime}\right)
\end{aligned}
$$


In view of Lemma 4.5 and Theorem 3.21, this proves the holomorphy of $\mathfrak{r}_{i}(\lambda)$.

4.10. Now we recall some results of Shimura $[16,17]$. Let $Q$ be the rational number field and let $K=Q(\zeta),[K: Q]=2 h$ as in 4.9. We denote by $\rho$ the complex conjugation. Let $\phi$ be a representation of $K$ by complex matrices of size $g$. We say that a triplet $\mathscr{P}=(A, \mathscr{C}, \theta)$ is a polarized abelian variety of type $\{K, \phi, \rho\}$ if the following conditions are satisfied:

(i) $A$ is an abelian variety of dimension $g$, defined over $C$.

(ii) $\theta$ is an isomorphism of $K$ into $\operatorname{End}_{Q}(A)$, and the representation of $\theta(x)$ for $x \in K$ by an analytic coordinate system of $A$ is equivalent to $\phi$.

(iii) $\mathscr{C}$ is an polarization of $A$; and the involution of $\operatorname{End}_{Q}(A)$ determined by $\mathscr{C}$ coincides with $\theta(x) \rightarrow \theta\left(x^{p}\right)$ on $\theta(K)$.

Let $\sigma_{1}, \ldots, \sigma_{h} ; \sigma_{1} \rho, \ldots, \sigma_{h} \rho$ be all the isomorphisms of $K$ into $C$ and let $r_{\nu}$ (resp. $s_{\nu}$ ) be the multiplicity of $\sigma_{\nu}\left(\right.$ resp. $\sigma_{\nu} \rho$ ) in $\phi$. In order to insure the existence of $\mathscr{P}$ of type $\{K, \phi, \rho\}$, the following relation should be satisfied:

$$
g=h\left(r_{\nu}+s_{\nu}\right) \quad(1 \leqq \nu \leqq h) .
$$

Put $u=g / h$.

Let $\mathscr{P}=(A, \mathscr{C}, \theta)$ be of type $\{K, \phi, \rho\}$. Take a complex torus $C^{\mathscr{B}} / D$ isomorphic to $A$, where $D$ is a lattice in $C^{g}$. We may choose the coordinate system of $C^{g}$ so that $\theta(a)$ is represented by the matrix $\phi(a)$ on $C$ for every $a \in K$. Then we find $u$ vectors $\mathfrak{x}_{1}, \ldots, \mathfrak{x}_{u}$ in $C^{g}$ such that

$$
Q D=\sum_{i=1}^{u} \dot{\phi}(K) \dot{\mathfrak{x}}_{i}
$$

For every $a=\left(a_{1}, \ldots, a_{u}\right)$ in $K^{u}$, put $x(a)=\sum_{i=1}^{u} \phi\left(a_{i}\right) \mathfrak{x}_{i}$. Then the mapping $a \rightarrow x(a)$ is an isomorphism of $K^{u}$ onto $Q D$. Let $M$ be the inverse image of $D$ by this mapping.

Let $E(x, y)$ be a non-degenerate Riemann form on $C^{g} / D$ corresponding to a basic polar divisor in $\mathscr{C}$. For each $i$ and $j$, the mapping $a \rightarrow E\left(\phi(a) \mathfrak{x}_{i}, \mathfrak{x}_{j}\right)$ is a $Q$-linear mapping of $K$ into $Q$. Hence there exists an element $t_{i j}$ of $K$ such that

$$
E\left(\phi(a) \mathfrak{x}_{i}, \mathfrak{r}_{j}\right)=\operatorname{tr}\left(a t_{i j}\right)
$$

where $\operatorname{tr}$ denotes the reduced trace of $K$ to $Q$. Put $T=\left(t_{i j}\right)$.

It is known by Shimura [16] that $\mathfrak{x}_{1}, \ldots, \mathfrak{x}_{u}, M$ and $T$ satisfy the following conditions : 


$$
C^{g}=\sum_{i=1}^{u} \phi\left(K \otimes_{Q} R\right) \mathfrak{x}_{i},
$$

(4.10.5) $T H^{\rho}$ is $\rho$-symmetric and $\rho$-positive, where $H=\left(h_{i j}\right)$ is a matrix, with entries in $K \otimes_{Q} R$ determined by $\sqrt{-1} \mathfrak{x}_{i}=\sum_{j=1}^{u} \phi\left(h_{i j}\right) \mathfrak{x}_{j} \quad(1 \leqq i \leqq u)$.

$$
\operatorname{tr}\left(M T M^{p}\right) \subset Z \text {. }
$$

Conversely the data $\left\{\mathfrak{x}_{1}, \ldots, \mathfrak{x}_{u} ; M ; T\right\}$ determine a polarized abelian variety of type $\{K, \phi, \rho\}$ if the above conditions are satisfied.

The above conditions $(4.10 .4,5)$ can be expressed in a more explicit form. First we write each vector $\mathfrak{x}_{i}$ in the form

$$
{ }^{t} \mathfrak{x}_{i}=\left({ }^{t} \mathfrak{x}_{i}^{1}, \ldots,{ }^{t} \mathfrak{x}_{i}^{v}, \ldots,{ }^{t} \mathfrak{x}_{i}^{h}\right)
$$

with $\mathfrak{x}_{i}^{\nu} \in C^{u}$. Define a matrix $X_{\vee}$ for each $\nu$ as follows. Put ${ }^{t} \mathfrak{x}_{i}^{\nu}=\left({ }^{t} u_{i 1}^{\nu},{ }^{t} v_{i 1}^{\nu}\right)$ with $u_{i 1}^{\nu} \in C^{r_{\nu}}, v_{i 1}^{\nu} \in C^{s_{\nu}}$ and

$$
X_{\nu}=\left[\begin{array}{l}
u_{11}^{\nu} \cdots u_{u 1}^{\nu} \\
\bar{v}_{11}^{\nu} \cdots \bar{v}_{u 1}^{\iota}
\end{array} \mid\right.
$$

Then the matrices $X_{\nu}$ for $1 \leqq \nu \leqq h$ determine the vectors $\mathfrak{x}_{1}, \ldots, \mathfrak{x}_{u}$ and conversely.

We see that the condition $(4.10 .4)$ is equivalent to

$$
\operatorname{det}\left(X_{\nu}\right) \neq 0 \quad(1 \leqq \nu \leqq h)
$$

and putting $T_{\nu}=\left(\sigma_{\nu}\left(t_{i j}\right)\right)$ the condition (4.10.5) is equivalent to the following condition :

$$
\sqrt{-1} \bar{X}_{\nu} T_{\nu}^{-1 t} X_{\nu}=\left[\begin{array}{cc}
\mathfrak{A}_{\nu} & 0 \\
0 & -\mathfrak{B}_{\nu}
\end{array}\right\rfloor
$$

- where $\mathfrak{A}_{\nu}$ and $\mathfrak{B}_{\nu}$ are positive hermitian matrices such that

$$
\mathfrak{U}_{\nu} \in M_{r_{\nu}}(C), \mathfrak{B}_{\nu} \in M_{s_{\nu}}(C),
$$

where $M_{m}(C)$ denotes the ring of matrices of degree $m$ with entries in $C$. Therefore our $T$ must satisfy the condition

$$
\sqrt{-1} T_{\nu}^{-1} \text { has the same signature as }\left[\begin{array}{cc}
I_{r_{\nu}} & 0 \\
0 & -I_{s_{\nu}}
\end{array}\right]
$$

for every $\nu$, where $I_{r}$ denotes the identity matrix of degree $r$.

Assuming (4.10.12) to be true, take, for each $\nu$, a matrix $W_{\nu}$ in. $M_{u}(C)$ such that

$$
W_{\nu}\left(\sqrt{ }-1 T_{\nu}^{-1}\right)^{t} \bar{W}_{\nu}=\left[\begin{array}{cc}
I_{r_{\nu}} & 0 \\
0 & -I_{s_{\nu}}
\end{array}\right]
$$


Put

$$
X_{\nu} \bar{W}_{\nu}^{-1}=\left[\begin{array}{ll}
\mathfrak{H}_{\nu} & \mathfrak{B}_{\nu} \\
\mathfrak{W}_{\nu} & \mathfrak{Y}_{\nu}
\end{array}\right] .
$$

Then $(4.10 .10)$ is equivalent to

$$
\begin{array}{ll}
\overline{\mathfrak{H}}_{\nu} \mathfrak{W}_{\nu}-\overline{\mathfrak{S}}_{\nu}^{t} \mathfrak{Y}_{\nu}=0, & \overline{\mathfrak{W}}^{t} \mathfrak{H}_{\nu}-\overline{\mathfrak{Y}}_{\nu}^{t} \mathfrak{B}_{\nu}=0 ; \text { and } \\
\overline{\mathfrak{H}}_{\nu}^{t} \mathfrak{H}_{\nu}-\overline{\mathfrak{V}}_{\nu}^{t} \mathfrak{W}_{\nu}, & \overline{\mathfrak{Y}}_{\nu}^{t} \mathfrak{Y}_{\nu}-\overline{\mathfrak{W}}_{\nu}^{t} \mathfrak{W}_{\nu} \text { are positive hermitian. }
\end{array}
$$

It follows that $\mathfrak{u}_{\nu}, \mathfrak{Y}_{v}$ are invertible, and $\mathfrak{u}_{\nu}^{-1} \mathfrak{B}_{\nu}={ }^{t}\left(\overline{\mathfrak{Y}_{\nu}^{-1} \mathfrak{B}_{\nu}}\right)$. Therefore, putting $z_{\nu}=\overline{\mathfrak{H}}_{\nu}^{-1} \mathfrak{B}_{\nu}={ }^{t}\left(\mathfrak{Y}_{\nu}^{-1} \mathfrak{B}_{\nu}\right)$, we observe that $(4.10 .15)$ is equivalent to

$$
I-z_{\nu}^{t} \bar{z}_{\nu} \text { is positive hermitian. }
$$

Let $\mathscr{H}_{\nu}$ be the space of all complex matrices $z$ with $r_{\nu}$ rows and $s_{\nu}$ columns such that $I-z_{\nu}^{t} \bar{z}_{\nu}$ is positive hermitian, and let

$$
\mathscr{B}(\phi)=\mathscr{H}_{1} \times \cdots \times \mathscr{H}_{h}=\mathscr{H}
$$

and let

$$
z=\left(z_{1}, \ldots, z_{h}\right)
$$

If we fix $T$ and $M$, then we get an analytic family of polarized abelian varieties of type $\{K, \phi, \rho\}$ parametrized by the points of $\mathscr{A}(\phi)$. We denote by $\Sigma(T, M)$ this family and by $\mathscr{P}_{z}$ the member of $\Sigma(T, M)$ determined by $z$, $T, M$. Summerizing, we write

$$
\Sigma=\Sigma(T, M)=\left\{\mathscr{P}_{z} \mid z \in \mathscr{H}\right\} .
$$

Let $\mathscr{P}=(A, \mathscr{C}, \theta)$ and $\mathscr{P} \prime=\left(A^{\prime}, \mathscr{C}^{\prime}, \theta^{\prime}\right)$ be polarized abelian varieties of type $\{K, \phi, \rho\}$. Let $\Gamma(T, M)=\left\{U \in M_{u}(K) \mid U T U^{\rho}=T, M U=M\right\} . \quad$ Then it is known that two members $\mathscr{P}_{z}$ and $\mathscr{P}_{z^{\prime}}$ of the same analytic family $\Sigma(T, M)$ are isomorphic if and only if $z=U\left(z^{\prime}\right)$ for an element $U$ of $\Gamma(T, M)$. For a proof, see Shimura [16]. Therefore the isomorphism classes of the members of $\Sigma$ are one to one correspondence with the points of the quotient space $\mathscr{H} / \Gamma(T, M)$. Now we denote sometimes $\Gamma(T, M)$ by $I$.

4.11. Now take a point $\lambda=\langle R, \sigma, \alpha\rangle$ in the generalized Teichmüller space $\Lambda\left(R_{0}, \sigma_{0}\right)$ defined in 4.3. Let $J(R(\lambda))$ be the jacobian variety of a representative $(R, \sigma)$ belonging to $\lambda$. Denote by $\theta(\zeta)$ be the automorphism of $J$ corresponding to $\sigma$. We see that $\zeta \rightarrow \theta(\zeta)$ can be extended naturally to an isomorphism $\theta$ of $Q(\zeta)$ into $\operatorname{End}_{\psi}(J)$. Let $\mathscr{C}$ be the canonical polarization of $J$, and $\rho$ the 
automorphism of $Q(\zeta)$ such that $\zeta^{\mathrm{p}}=\zeta^{-1}$. The involution of $\operatorname{End}_{Q}(J)$ determined by $\mathscr{C}$ gives the automorphism $\theta(a) \rightarrow \theta\left(a^{p}\right)$ on $\theta(Q(\zeta))$. In this way we get a polarized abelian variety of type $\{Q(\zeta), \phi, \rho\}$ in the sence of 4.10 , for a representation $\phi$ of degree $g$ defined in 4.4 .

We see that if $(R, \sigma)$ and $\left(R^{\prime}, \sigma^{\prime}\right)$ belong to the same element $\langle R, \sigma\rangle$, then corresponding polarized abelian varieties $\mathscr{P}$ and $\mathscr{P}$ are isomorphic and vice versa. As seen above, to every point $\lambda$ in $A\left(R_{0}, \sigma_{0}\right)$ there corresponds a polarized jacobian variety $\mathscr{P}$, and furthermore we see that all these varieties belong to the same type $\{Q(\zeta), \phi, \rho\}$. As we see in $4.4-4.8$, for an arbitrary point $\lambda^{*}$ in $\Lambda\left(R_{0}, \sigma\right)$ there exists a neighborhood $V\left(\lambda^{*}\right)$ such that we can define a data $\left\{\mathfrak{x}_{1}(\lambda), \ldots, \mathfrak{x}_{u}(\lambda) ; M(\lambda) ; T(\lambda)\right\}$ corresponding to any point $\lambda$ in $V\left(\lambda^{*}\right)$. That is, we define the vectors by $(4.9 .4)$, and we define $M(\lambda)$ by

$$
\begin{aligned}
M(\lambda)=\left\{\left(a_{1}, \ldots, a_{u}\right) \in K^{u} \mid \varphi_{*}\left(a_{1}, \lambda\right) Z_{1}(\lambda)\right. & +\cdots \\
& \left.+\varphi_{*}\left(a_{u}, \lambda\right) Z_{u}(\lambda) \in H_{1}(R(\tau), Z)\right\} .
\end{aligned}
$$

As for $T(\lambda)$, it is defined by $(4.10 .3)$ :

$$
E\left(\phi(a) \mathfrak{x}_{i}(\lambda), \mathfrak{x}_{j}(\lambda)\right)=\operatorname{tr}\left(a t_{i j}(\lambda)\right) .
$$

It is easy to see that this data is nothing but the data defined in (4.10).

Now let the data corresponding to a point $\lambda^{*} \in \Lambda\left(R_{0}, \sigma_{0}\right)$ be $\left\{\mathfrak{r}_{1}^{*}, \ldots, \mathfrak{x}_{\mathfrak{u}}^{*}\right.$; $\left.M^{*} ; T^{*}\right\}$. Then we obtain the following lemma.

4.12. Lemma. Let the data corresponding to a point $\lambda$ in a sufficiently small neighborhood $V\left(\lambda^{*}\right)$ be $\left\{\mathfrak{x}_{1}(\lambda), \ldots, \mathfrak{x}_{u}(\lambda) ; M(\lambda) ; T(\lambda)\right\}$. Then we have $M(\lambda)=M^{*}$, and $T(\lambda)=T^{*}$.

Proof. If

$$
\varphi_{*}\left(a_{1}, \lambda^{*}\right) Z_{1}\left(\lambda^{*}\right)+\cdots+\varphi_{*}\left(a_{u}, \lambda^{*}\right) Z_{u}\left(\lambda^{*}\right) \in H_{1}\left(R\left(\tau^{*}\right), Z\right),
$$

then we see easily that

$$
\varphi_{*}\left(a_{1}, \lambda\right) Z_{1}(\lambda)+\cdots+\varphi_{*}\left(a_{u}, \lambda\right) Z_{u}(\lambda) \equiv H_{1}(R(\tau), Z)
$$

since we have $f_{*} \varphi_{*}\left(a, \lambda_{*}\right)=\varphi_{*}(a, \lambda) f_{*}$. Therefore we can conclude that

$$
M(\lambda)=M\left(\lambda^{*}\right)=M^{*}
$$

for all $\lambda \in V\left(\lambda^{*}\right)$.

As for $T(\lambda)$, we see that for all $x_{i}, y_{j} \in K$, 


$$
\begin{aligned}
\operatorname{tr}\left(\sum_{i j} x_{i} t_{i j}(\lambda) y_{j}\right)=E\left(\phi\left(x_{1}\right) \mathfrak{x}_{1}(\lambda)+\cdots+\phi\left(x_{u}\right) \mathfrak{x}_{u}(\lambda), \phi\left(y_{1}\right) \mathfrak{x}_{1}(\lambda)+\cdots+\phi\left(y_{u}\right) \mathfrak{x}_{u}(\lambda)\right) \\
\quad=K I\left(\varphi_{*}\left(x_{1}\right) Z_{1}(\lambda)+\cdots+\varphi_{*}\left(x_{u}\right) Z_{u}(\lambda), \varphi_{*}\left(y_{1}\right) Z_{1}(\lambda)+\cdots+\varphi_{*}\left(y_{u}\right) Z_{u}(\lambda)\right) \\
\quad=K I\left(\varphi_{*}\left(x_{1}\right) Z_{1}\left(\lambda^{*}\right)+\cdots+\varphi_{*}\left(x_{u}\right) Z_{u}\left(\lambda^{*}\right), \varphi_{*}\left(y_{1}\right) Z_{1}\left(\lambda^{*}\right)+\cdots+\varphi_{*}\left(y_{u}\right) Z_{u}(\lambda)\right) \\
\quad=E\left(\phi\left(x_{1}\right) \mathfrak{x}_{1}\left(\lambda^{*}\right)+\cdots+\phi\left(x_{u}\right) \mathfrak{x}_{u}\left(\lambda^{*}\right), \phi\left(y_{1}\right) \mathfrak{x}_{1}\left(\lambda^{*}\right)+\cdots+\phi\left(y_{u}\right) \mathfrak{x}_{u}\left(\lambda^{*}\right)\right) \\
\quad=\operatorname{tr}\left(\sum_{i, j} x_{i} t_{i j}\left(\lambda^{*}\right) y_{j}\right) .
\end{aligned}
$$

Here $K I$ means the Kronecker index. From this we can conclude that

$$
T(\lambda)=T\left(\lambda^{*}\right)=T^{*}
$$

for all $\lambda \in V\left(\lambda^{*}\right)$.

By the same reason as above we see that $M(\lambda), T(\lambda)$ are constants for all $\lambda \in \Lambda$.

Moreover we see that $\mathfrak{x}_{1}(\lambda), \ldots, \mathfrak{x}_{u}(\lambda)$ are holomorphic in $V\left(\lambda^{*}\right)$, by 4.9 .

Now we can state an important theorem as follows.

4.13. TheOREm. Let $\lambda_{0}=\left\langle R_{0}, \sigma_{0}, \alpha_{0}\right\rangle$ be the origin of the generalized Teichmüller space $\Lambda\left(R_{0}, \sigma_{0}\right)$ as in 4.3 , and let $\lambda=\langle R, \sigma, \alpha\rangle$ be an arbitrary element of $\Lambda$. We get from these $(R, \sigma)$ polarized abelian varieties of type $\{K, \phi, \rho\}$ for a certain representation $\phi$ as in 4.11. Let $\mathscr{H}$ be the corresponding symmetric domain defined by (4.10.17). Then there exists a holomorphic mapping $w(\lambda)$ of $\Lambda\left(R_{0}, \sigma_{0}\right)$ into $\mathscr{I}$ such that the corresponding jacobian variety of $R(\tau)$, where $\tau=\iota(\lambda)$, defined as in 4.11 is isomorphic to a polarized abelian variety $\mathscr{P}_{w(\lambda)}$ defined in 4.10 .

Proof. As we see in 4.11 and Lemma 4.12 for an arbitrary point $\lambda^{*} \in \Lambda$, the data corresponding to a point $\lambda$ in a sufficiently small neighborhood $V\left(\lambda^{*}\right)$ can be denoted as $\left\{x_{1}(\lambda), \ldots, x_{u}(\lambda) ; M\left(\lambda_{0}\right) ; T\left(\lambda_{0}\right)\right\}$. Here we remark that $\mathfrak{x}_{1}(\lambda), \ldots, \mathfrak{x}_{\mathfrak{u}}(\lambda)$ are holomorphic and we write $M\left(\lambda_{0}\right), T\left(\lambda_{0}\right)$ by $M, T$ respectively.

We can correspond to this data a point $z \in \mathscr{L}$ as in 4.10 . That is, we have a mapping $z(\lambda)$ of $V\left(\lambda^{*}\right)$ into $\mathscr{A}$. If we put

$$
z(\lambda)=\left(z_{1}(\lambda), \ldots, z_{u}(\lambda)^{\prime}\right)
$$

then $z_{\nu}(\lambda)$ is equal to ${ }^{t}\left(\mathfrak{Y}_{\nu}^{-1}(\lambda) \mathfrak{B}_{\nu}(\lambda)\right)(z=1, \ldots, h)$ as in 4.10 , which is holomorphic in $V\left(\lambda^{*}\right)$. In fact, $\mathfrak{Y}_{\nu}(\lambda)$ and $\mathfrak{W}_{\nu}(\lambda)$ are holomorphic in $V\left(\lambda^{*}\right)$ and $\operatorname{det}\left(Y_{\nu}(\lambda)\right)$ is not equal to zero in $V\left(\lambda^{*}\right)$. Thus we see that there exists a holomorphic mapping $z(\lambda)$ in a neighborhood $V\left(\lambda^{*}\right)$ for every point $\lambda^{*} \in \Lambda\left(R_{0}, \sigma_{0}\right)$. In this way we can find an open covering $\mathscr{V}=\{V\}$ of $\Lambda$ and a holomorphic mapping $z_{V}: V \rightarrow \mathscr{H}$, for each $V \in \mathscr{V}$, with the above property of $V\left(\lambda^{*}\right)$ and 
$z(\lambda)$. We may assume that $V \cap V^{\prime}$ is connected if $V$ and $V^{\prime}$ are members of $\mathscr{V}$ such that $V \cap V^{i} \neq \phi$.

Let $V, V^{\prime}$ be the neighborhoods and let

$$
z: V \rightarrow \mathscr{H} ; z^{\prime}: V^{\prime} \rightarrow \mathscr{H}
$$

be holomorphic mappings. Then for every $\lambda \in V \cap V^{\prime}$, we have

$$
z(\lambda)=U_{\lambda}\left(z^{\prime}(\lambda)\right)
$$

for an element $U_{\lambda}$ of $\Gamma(T, M)$, since $\mathscr{P}_{z(\lambda)}$ and $\mathscr{P}_{z^{\prime}(\lambda)}$ are isomorphic (see 4.10).

Now as we see in [16] for an arbitrary $U \in \Gamma(T, M), U(z)$ is a rational function in the coordinates of $z$. Let $k_{1}$ be an extension field of $Q$ by the adjunction of the set which consists of all the coefficients of $U(z)$ for all $U \in \Gamma(T, M)$.

Since $I^{\prime}(T, M)$ is a discrete subgroup of $G(T)=\left\{U \in M_{u}\left(K \otimes_{Q} R\right) \mid U T^{t} \bar{U}=T\right\}$, $k_{1}$ has at most countable elements. Therefore we can take a generic point $\lambda_{1}$ of $V \cap V^{\prime}$ for $\left\{z(\lambda), z^{\prime}(\lambda)\right\}$ over $k_{1}$ in the sense of Shimura. Put $U=U_{\lambda_{1}}$, then we have

$$
z\left(\lambda_{1}\right)=U\left(z^{\prime}\left(\lambda_{1}\right)\right)
$$

Since $\lambda_{1}$ is generic over $k_{1}$, we have

$$
z(\lambda)=U\left(z^{\prime}(\lambda)\right)
$$

for every $\lambda \in V \cap V^{\prime}$. Putting

$$
w(\lambda)= \begin{cases}z(\lambda) & \text { in } \lambda \in V \\ U\left(z^{\prime}(\lambda)\right) & \text { in } \lambda \in V^{\prime}\end{cases}
$$

we can prolong the holomorphic mapping $z(\lambda): V \rightarrow \not{A}$ to a holomorphic mapping $w(\lambda): V \cup V^{\prime} \rightarrow \mathscr{L}$.

Now starting with the origin $\lambda_{0}$ we continue the above process to all points in $\Lambda\left(R_{0}, \sigma_{0}\right)$. Since $A$ is simply connected, finally we can obtain a holomorphic mapping of $\Lambda$ into $\mathscr{H}$ :

$$
w: \Lambda \rightarrow \mathscr{H}
$$

such that $w(\lambda)=U_{V}\left(z_{V}(\lambda)\right)$ in $V$, with an element $U_{V}$ of $\Gamma(T, M)$. Here $z_{v}$ denotes the original holomorphic mapping of $V \rightarrow \mathscr{H}$.

Thus the data $\left\{r_{1}(\lambda), \ldots, x_{u}(\lambda) ; M ; T\right\}$ corresponding to a point $\lambda$ in $\Lambda$ determines a point $w(\lambda)$ in $\mathscr{H}$ up to $\Gamma$-equivalence. We see that this point 
$w(\lambda)$ can be represented by $w(\lambda)=U_{V}\left(z_{V}(\lambda)\right)$ in $V\left(\lambda^{*}\right)$.

The second assertion of the theorem is an immediate consequence of our definition.

\section{$\S 5$. Holomorphy of parameters}

5.1. By proposition 4.2 we see that $\Omega\left(0, n,\left\{\nu_{1}, \ldots, \nu_{r}\right\}\right)$ is a family of $\langle R, \sigma\rangle$ where $R$ is defined by

$$
y^{n}=\left(x-a_{1}\right)^{n t_{1}} \cdots\left(x-a_{s}\right)^{m_{s}}, n+\sum_{i=1}^{s} m_{i}, r=s+1,
$$

as (4.3.1), and $\sigma$ is an automorphism of $R$ such that $(x, y) \rightarrow(x, \zeta y), \zeta=e^{2 \pi i / n}$. We shall denote this $R$ by $R_{a}$ and the $\sigma$ by $\sigma_{a}$. Then $\left(R_{a}, \sigma_{a}\right)$ defines a point $\lambda \in \Lambda\left(R_{0}, \sigma_{0}\right)$.

As in 4.11 , we get a polarized jacobian variety $(J, \mathscr{C}, \theta)$ of type $\{K, \phi, \rho\}$ from $(R, \sigma)$. Let $H$ be the corresponding symmetric domain. Let $T, M, T(T, M)$, $\Sigma=\Sigma(T, M)=\left\{\mathscr{P}_{z} \mid z \in H\right\}$ be as in 4.10. We shall investigate the holomorphy of parameters $a_{1}, \ldots, a_{s}$ with respect to the complex structure of $\Lambda\left(R_{0}, \sigma_{0}\right)$. Suppose that $n>3$ or $s>3$.

5.2. Lemma [18]. Suppose that either $\operatorname{dim} H>1$, or $H / \Gamma(T, M)$ is compact. Let $k$ be the algebraic number field in [18. Th. 5.1]. Then one can define a variety $\widetilde{\mathfrak{B}}$ and assign, to every $\mathscr{P}$ of type $\{Q(\zeta), \phi, \rho\}$, exactly one point $\tilde{\mathfrak{b}}(\mathscr{P})$ of $\widetilde{\mathfrak{B}}$ so that the following conditions are satisfied.

(5.2.1) $\tilde{\mathfrak{B}}$ is defined over $k$.

(5.2.2) $\tilde{\mathfrak{v}}(\mathscr{P})=\tilde{\mathfrak{v}}\left(\mathscr{P}^{\prime}\right)$ if and only if $\mathscr{P}$ is isomorphic to $\mathscr{P}$ '.

(5.2.3) Let $\mathscr{P}=(A, \mathscr{C}, \theta)$ be a member of the family $\Sigma$ of type $\{Q(\zeta), \phi, \rho\}$ difined over a field $K$. Suppose that $\mathscr{P} 1$ is a specialization of $\mathscr{P}$ over $K$, then $(\tilde{\mathfrak{D}}(\mathscr{P}), \mathscr{P} \prime)$ is a specialization of $(\tilde{\mathfrak{v}}(\mathscr{P}), \mathscr{P})$ over $K$.

(5.2.4) $k(\tilde{\mathfrak{v}}(\mathscr{P}))$ is the field of moduli of $\mathscr{P}$.

(5.2.5) There exists a holomorphic mapping $\varphi$ of $\mathscr{H}$ onto $\tilde{\mathfrak{B}}$, which induces an isomorphism of $\mathscr{H} / \Gamma(T, M)$ onto $\widetilde{\mathfrak{B}}$, and such that $\tilde{\mathfrak{b}}\left(\mathscr{P}_{w}\right)=\varphi(w)$ for every member $\mathscr{P}_{w}$ of $\Sigma$.

5.3. As we see in 4.13 there exists a holomorphic mapping $w$ of $A$ into $\mathscr{A}$. Let $\varphi$ be the natural mapping of $H$ onto $\mathfrak{B}=\mathscr{A} / \Gamma(T, M)$. Then, of course, the mapping $\varphi \cdot w$ of $\Lambda$ into $\mathfrak{B}$ is holomorphic.

Let $S=\left\{\left(x_{1}, \ldots, x_{s}\right) \in C^{s} \mid x_{i} \neq x_{j}\right.$ if $\left.i \neq j\right\}$. Then $S$ is a Zariski open subset 
in $C^{s}$. Let $\left(b_{1}, \ldots, b_{s}\right)$ be a generic point of $S$ over the field $k \supset Q(\zeta)$. Let $\left(R_{b}, \sigma_{b}\right)$ be the point corresponding to $\left(b_{1}, \ldots, b_{s}\right)$. Then $\left(R_{b}, \sigma_{b}\right)$ is defined over the field $k\left(b_{1}, \ldots, b_{s}\right)$. Let $\lambda_{1} \in \Lambda$ be a point corresponding to $\left(b_{1}, \ldots, b_{s}\right)$. In the equivalence class of $\Omega$ corresponding to $\lambda_{1}$ there exists an element $\left(R_{b}, \sigma_{b}\right)$ which is defined over $k\left(b_{1}, \ldots, b_{s}\right)$. Therefore we have an element of $\Sigma(T, M)$ which corresponds to $\left(R_{b}, \sigma_{b}\right)$ and which is isomorphic to $\mathscr{P}_{w\left(\lambda_{1}\right)}$, and which is defined over $k\left(b_{1}, \ldots, b_{s}\right)$. Then we have

$$
k\left(\varphi\left(w\left(\lambda_{1}\right)\right)\right) \subset k\left(b_{1}, \ldots, b_{s}\right) .
$$

In fact, by the properties of $\widetilde{\mathfrak{B}}$ we see that $k\left(\tilde{\mathfrak{b}}\left(\mathscr{P}_{\left.w_{1} \lambda_{1}\right)}\right)\right)$ is the field of moduli of $\mathscr{P}_{w\left(\lambda_{1}\right)}$, and $\tilde{\mathfrak{v}}\left(\mathscr{P}_{w\left(\lambda_{1}\right)}\right)=\varphi\left(w\left(\lambda_{1}\right)\right)$. Hence we can define a rational mapping $\psi: S \rightarrow \widehat{\mathfrak{B}}$, defined over $k$, by

$$
\psi\left(b_{1}, \ldots, b_{s}\right)=\varphi\left(w\left(\lambda_{1}\right)\right) .
$$

Now let $\lambda \in \Lambda$ be"a point corresponding to a point $\left(a_{1}, \ldots, a_{s}\right) \in S$, then obviously $\left(R_{a}, \sigma_{a}\right)$ is the specialization of $\left(R_{b}, \sigma_{b}\right)$ over the specialization $\left(a_{1}, \ldots, a_{s}\right)$ of $\left(b_{1}, \ldots, b_{s}\right)$ with reference to $k$.

5.4. Now we shall construct an analytic isomorphism of a Riemann surface $R$ of genus $g$ into a projective space.

Let $D=\sum_{P \in R} n_{P} P$ be a divisor such that $\operatorname{deg} D>2 g$, where the $n_{P}$ are integers and zeros except for finite numbers of them. Let $L(D)$ be the vector space of meromorphic functions $f$ of $R$ such that $o_{P}(f) \geqq-n_{R}$ for all $P \in R$, where $o_{P}$ is the order of $f$ at $P$. Then we obtain the following well-known lemma [15].

5.5. Lemma. Let $f_{0}, \ldots, f_{N}$ be a basis of $L(D)$. For all $P \in R$, let $F(P)$ be the point of the projective space $P_{N}$ of homogeneous coordinates $f_{0}(P), \ldots, f_{N}(P)$. The mapping $F$ is an analytic isomorphism of $R$ onto a non-singular subvariety of $P_{s}$ and the divisor $D$ is equivalent to a hyper-piane section of $F(R)$.

5.6. Now again we take a generic point $\left(b_{1}, \ldots, b_{s}\right)$ of $S$ over the field $k$. Let $D_{b}$ be a divisor defined as in 5.4 , for the Riemann surface corresponding to $\left(b_{1}, \ldots, b_{s}\right)$. Let $D_{a}$ be the specialization of $D_{b}$ of over $\left(b_{1}, \ldots, b_{s}\right) \rightarrow\left(a_{1}, \ldots, a_{s}\right)$ with reference to $k$ and let $L\left(D_{j}\right), L\left(D_{a}\right)$ be the corresponding vector spaces defined as in 5.4 .

By a theorem of Koizumi-Shimura [11] we can find a basis $\left\{f_{0}, \ldots, f_{N}\right\}$ of $L\left(D_{b}\right)$ such that the specialization $\left\{f_{0}^{\prime}, \ldots, f_{N}^{\prime}\right\}$ of $\left\{f_{0}, \ldots, f_{N}\right\}$ over $R_{b} \rightarrow R_{a}$ 
is a basis of $L\left(D_{a}\right)$. Then we construct $C_{a}$ by means of the basis $\left\{f_{0}^{\prime}, \ldots, f_{N}^{\prime}\right\}$ following Lemma 5.5. Let $C^{\prime}$ be a specialization of $C_{b}$ over $\left(b_{1}, \ldots, b_{s}\right) \rightarrow$ $\left(a_{1}, \ldots, a_{s}\right)$ with reference to $k$. Since the complete system $|D|$ has no fixed components we have $\operatorname{deg}\left(D_{a}\right)=\operatorname{deg}\left(C_{a}\right), \operatorname{deg}\left(D_{b}\right)=\operatorname{deg}\left(C_{b}\right)$, and we have obviously $\operatorname{deg}\left(D_{a}\right)=\operatorname{deg}\left(D_{b}\right)$. On the other hand we see that $C^{\prime}-C_{a}$ is positive, i.e., $C^{\prime}>C_{a}$. However we have $\operatorname{deg}\left(C^{\prime}\right)=\operatorname{deg}\left(C_{b}\right)=\operatorname{deg}\left(C_{a}\right)$. Therefore $C^{\prime}=C_{a}$.

Now let $\tau_{b}$ be the isomorphism of the curve $C_{b}$ induced by $\sigma_{b}$, and let $\left(C_{b}, \tau_{b}\right)$ be the couple corresponding to $\left(R_{b}, \sigma_{b}\right)$ and $\left(C_{a}, \tau_{a}\right)$ be the couple corresponding to $\left(R_{a}, \sigma_{a}\right)$. We have seen that $C_{a}$ is the specialization of $C_{b}$ over $\left(b_{1}, \ldots, b_{s}\right)$ $\rightarrow\left(a_{1}, \ldots, a_{s}\right)$ with reference to $k$. Furthermore we see that $\tau_{a}$ is the specialization of $\tau_{b}$ over $\left(b_{1}, \ldots, b_{s}\right) \rightarrow\left(a_{1}, \ldots, a_{s}\right)$ with reference to $k$.

Thus we obtain the following lemma.

5.7. Lemma. Notations being as above, $\left(C_{a}, \tau_{a}\right)$ is the unique specialization of $\left(C_{b}, \tau_{b}\right)$ over $\left(b_{1}, \ldots, b_{s}\right) \rightarrow\left(a_{1}, \ldots, a_{s}\right)$ with reference to $k$.

Now we have moreover the following lemma.

5.8. Lemma. Let $\left(J_{b}, \mathscr{C}_{b}, \theta_{b}\right)$ and $\left(J_{a}, \mathscr{C}_{a}, \theta_{a}\right)$ be the polarized jacobian varieties of type $(K, \phi, \rho)$ corresponding to $\left(R_{b}, \sigma_{b}\right)$ and $\left(R_{a}, \sigma_{a}\right)$ respectively. Then $\left(J_{a}, \mathscr{C}_{a}, \theta_{a}\right)$ is the unique specialization of $\left(J_{b}, \mathscr{C}_{b}, \theta_{b}\right)$ over $\left(b_{1}, \ldots, b_{s}\right) \rightarrow\left(a_{1}, \ldots, a_{s}\right)$ with reference to $k$.

Proof. By Lemma 5.6 we see that if $\left(R_{b}\right) \rightarrow\left(R_{a}\right)$ over $\left(b_{1}, \ldots, b_{s}\right) \rightarrow$ $\left(a_{1}, \ldots, a_{s}\right)$ with reference to $k$, then

$$
\left(\left(C_{b}, \tau_{b}\right),\left(R_{b}, \sigma_{b}\right)\right) \rightarrow\left(\left(C_{a}, \tau_{a}\right),\left(R_{a}, \sigma_{a}\right)\right)
$$

over $\left(R_{b}, \sigma_{b}\right) \rightarrow\left(R_{a}, \sigma_{a}\right)$.

Then by the compatibility theorem of Igusa [10]

$$
\left(C_{b}, \tau_{b} ; J_{b}, \mathscr{C}_{b}\right) \rightarrow\left(C_{a}, \tau_{a} ; J_{a}, \mathscr{C}_{a}\right)
$$

over $\left(b_{1}, \ldots, b_{s}\right) \rightarrow\left(a_{1}, \ldots, a_{s}\right)$ with reference to $k$.

Furthermore, we see that $\theta_{b} \rightarrow \theta_{a}$ over (5.8.2).

5.9. Now we construct a specialization $\left(a_{1}, \ldots, a_{s} ; c\right)$ of $\left(b_{1}, \ldots, b_{s}\right.$; $\left.\psi\left(b_{1}, \ldots, b_{s}\right)\right)$ over $k$.

By Lemma 5.8 we see that $\mathscr{P}_{w(\lambda)}$ is the unique specialization of $\mathscr{P}_{w\left(\lambda_{1}\right)}$ 
over the specialization $\left(b_{1}, \ldots, b_{s} ; \psi\left(b_{1}, \ldots, b_{s}\right)\right) \rightarrow\left(a_{1} \ldots, a_{s} ; c\right)$. Therefore by Lemma $5.2\left(a_{1}, \ldots, a_{s} ; c ; \mathscr{P}_{w(\lambda)} ; \varphi(w(\lambda))\right)$ is a specialization of $\left(b_{1}, \ldots, b_{s} ; \phi\left(b_{1}, \ldots, b_{s}\right) ; \mathscr{P}_{w\left(\lambda_{1}\right)} ; \varphi(w(\lambda))\right)$. Since $\psi\left(b_{1}, \ldots, b_{s}\right)=\varphi\left(w\left(\lambda_{1}\right)\right)$, we see that $c=\varphi(w(\lambda))$.

In fine, we obtain the following facts.

(I) $c$ is the unique specialization of $\psi\left(b_{1}, \ldots, b_{s}\right)$ over the specialization $\left(b_{1}, \ldots, b_{s}\right) \rightarrow\left(a_{1}, \ldots, a_{s}\right)$ with reference to $k$.

(II) If $\left(a_{1}, \ldots, a_{s}\right)$ corresponds to $\lambda$, then the unique specialization $c=\psi\left(a_{1}, \ldots, a_{s}\right)$ is equal to $\varphi(w(\lambda))$.

5.10. Theorem. Notations being as above, there exists a rational mapping $\psi: S \rightarrow \widetilde{\mathfrak{B}}$ with the following properties.

(5.10.1) $\psi$ is a morphism, i.e., everywhere defined on $S$.

(5.10.2) $\psi\left(a_{1}, \ldots, a_{s}\right)=\varphi(w(\lambda))$ if $\lambda$ corresponds to $\left(a_{1}, \ldots, a_{s}\right)$ in the above sense.

§ 6. Special case: $\Omega(0,7,\{1,1,1,2\})$

6.1. Now we give our attention to the case where $\Lambda\left(R_{0}, \sigma_{0}\right)$ is a 1 -dimentional manifold and $g^{\prime}=0$ in $\Omega\left(g^{\prime}, n,\left\{\nu_{i}\right\}\right)$, over which we construct $\Lambda\left(R_{0}, \sigma_{0}\right)$. Then by Theorem 3.21 we see that the space $\Lambda\left(R_{0}, \sigma_{0}\right)$ is holomorphically equivalent to the upper-half plane. For example, if we put $g^{\prime}=0, n=7$, $\left\{\nu_{i}\right\}=\{1,1,1,2\}$, then $\Omega\left(g^{\prime}, n,\left\{\nu_{i}\right\}\right)$ is a family of $\langle R, \sigma\rangle$, where $R$ is a Riemann surface defined by the equation:

$$
y^{7}=x(x-1)(x-\alpha)
$$

and $\sigma$ is an automorphism such that $(x, y) \rightarrow(x, \zeta y), \zeta=e^{2 \pi i / 7}$.

In general, by 3.4 we see that the equation of the Riemann surface is

$$
y^{n}=x^{m_{1}}(x-1)^{m_{2}}(x-\alpha)^{m_{3}},
$$

where $m_{i} \nu_{i} \equiv 1(\bmod n)$ for $i=1,2,3$. The genus of $(6.1 .2)$ is equal to $n-1$.

In the present paper, we treated the moduli of $\Omega$ only at the special case (6.1.1). However it would be interesting to study the analogy of the theory of elliptic functions in our case.

Now we reappear the calculation in 4.10 for our case : $\Omega(0,7,\{1,1,1,2\})$.

6,2. We see that the matrix $\phi(\zeta)$ is of the form: 


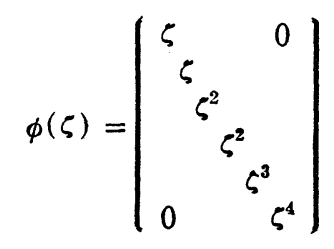

Furthermore we see that $g=6, h=3$, and $u=2$ in (4.10.1), and $r_{1}=r_{2}=2$, $s_{1}=s_{2}=0, r_{3}=s_{3}=1$.

Put

$$
\begin{aligned}
& { }^{t} \mathfrak{x}_{1}=\left(u_{11}, u_{31} ; u_{31}, u_{41} ; u_{51} ; u_{61}\right) \\
& { }^{t} \mathfrak{x}_{2}=\left(u_{12}, u_{22} ; u_{32}, u_{42} ; u_{32} ; u_{62}\right) .
\end{aligned}
$$

Then the period matrices $X_{\nu}, \nu=1,2,3$ are as follows:

$$
X_{1}=\left(\begin{array}{ll}
u_{11} & u_{12} \\
u_{21} & u_{22}
\end{array}\right), \quad X_{2}=\left(\begin{array}{ll}
u_{31} & u_{32} \\
u_{11} & u_{32}
\end{array}\right), \quad X_{3}=\left(\begin{array}{ll}
u_{51} & u_{52} \\
\bar{u}_{61} & \bar{u}_{62}
\end{array}\right) .
$$

We have following relations for the matrices as $T_{\nu}$ in 4.10 :

$$
\begin{aligned}
& W_{1}\left(\sqrt{-1} T_{1}^{-1}\right)^{t} \bar{W}_{1}=W_{2}\left(\sqrt{-1} T_{2}^{-1}\right)^{t} \bar{W}_{2}=\left(\begin{array}{ll}
1 & 0 \\
0 & 1
\end{array}\right) . \\
& W_{3}\left(\sqrt{-1} T_{3}^{-1}\right)^{t} \bar{W}_{3}=\left(\begin{array}{rr}
1 & 0 \\
0 & -1
\end{array}\right) .
\end{aligned}
$$

applying suitable matrices $W_{i}$ to $T_{\nu}$.

Now in our case we have only to investigate (6.2.4). Put

$$
X_{3} \bar{W}_{3}^{-1}=\left(\begin{array}{ll}
u_{51} & u_{52} \\
\bar{u}_{61} & \bar{u}_{62}
\end{array}\right)\left(\begin{array}{ll}
\bar{\alpha} & \bar{\beta} \\
\bar{\gamma} & \bar{\delta}
\end{array}\right)=\left(\begin{array}{ll}
u & v \\
w & y
\end{array}\right)
$$

Then we have $u / v=(\overline{w / y})$, and

$$
\bar{X}_{3} \sqrt{-1} T_{3}^{-1 t} X_{3}=\left(\begin{array}{cc}
|u|^{2}-|v|^{2} & 0 \\
0 & |w|^{2}-|y|^{2}
\end{array}\right)
$$

We must notice here that $|u|^{2}-|v|^{2}>0,|w|^{2}-|y|^{2}<0$. Thus putting $z=(u / v)$, we observe that $1-z \bar{z}>0$.

Now we can see that

$$
\begin{aligned}
z & =(\overline{w / y}) \\
& =\left(\alpha\left(u_{61} / u_{62}\right)+\gamma\right) /\left(\beta\left(u_{61} / u_{62}\right)+\delta\right) \\
& =\frac{\alpha\left(\int_{z_{1}} y^{-3} d x / \int_{z_{2}} y^{-3} d x\right)+\gamma}{\beta\left(\int_{z_{1}} y^{-3} d x / \int_{z_{2}} y^{-3} d x\right)+\delta}
\end{aligned}
$$


Here $Z_{1}, Z_{2}$ are vectors defined as in (4.9), and $\int_{Z_{i}}(x(x-1)(x-\alpha))^{-3 / \pi} d x$ are hypergeometric functions in $\alpha$.

It must be interesting that in our case both $A$ and $\mathscr{H}$ are the upperhalf plane and the holomorphic mapping $z(\lambda)$ is exactly a ratio of the periods of the differentials of the first kind of Riemann surfaces, corresponding to $\lambda$. Moreover we must remark that in our case $\mathscr{H} / \Gamma(T, M)$ is compact (cf. [17]).

We shall study some elementary properties of the parameter $\alpha$.

6.3. Let $R^{\prime}$ be another Riemann surface defined by the equation:

$$
y^{\prime 7}=x^{\prime}\left(x^{\prime}-1\right)\left(x^{\prime}-\beta\right) \quad(\beta \neq 0,1) .
$$

Then we get a polarized abelian variety $\mathscr{P}$ ' of type $\{Q(\zeta), \phi, \rho\}$ by $R^{\prime}$ in the same way as in 6.1.

We recall that $\mathscr{P}$ is isomorphic to $\mathscr{P}^{\prime}$ if and only if there exists a holomorphic bijection $f: R^{\prime}$ such that $f_{\sigma}=\sigma^{\prime} f(\mathrm{cf}$. [17]).

We see also that if $(R, \sigma)$ and $\left(R^{\prime}, \sigma^{\prime}\right)$ belong to the same $\langle R, \sigma\rangle$ then

$$
x^{\prime}=a x+b, y^{\prime}=c y \text {. }
$$

Here $a, b$, and $c$ are complex numbers such that

$$
\begin{aligned}
& a=1, b=0 ; a=1 / \alpha, b=0 ; a=-1, b=1 ; a=-1 / \alpha, b=1, \\
& a=-\alpha /(1-\alpha), b=1 /(1-\alpha) ; a=-\alpha /(1-\alpha), b=\alpha /(1-\alpha),
\end{aligned}
$$

and $c=1$. Therefore $(R, \sigma)$ and $\left(R^{\prime}, \sigma^{\prime}\right)$ are equivalent if and only if $\beta=\alpha$, $\beta=1 / \alpha, \beta=1-\alpha, \beta=1 /(1-\alpha), \beta=(\alpha-1) / \alpha, \beta=\alpha /(\alpha-1)$.

6.4. Let $S^{\prime}$ be a Riemann sphere punctured at 0,1 , and infinity $\infty$. Let $G$ be the group of the anharmonic ratio. Then the quotient space $\tilde{S}=S^{\prime} / G$ is a Riemann surface and there exists a one-to-one correspondence between the space of moduli $\Omega$ and $\widetilde{S}$. $\Omega$ has a complex structure induced from $\Lambda\left(R_{0}, \sigma_{0}\right)$ and $\widetilde{S}$ has a natural complex structure. Our problem is to investigate the analyticity of the correspondence.

6.5. We consider the above problem in a little more simple form; that is, we consider the Riemann surface defined by the equation:

$$
y^{7}=x^{3}+x+p
$$

where $p$ is a complex number different from $-2 i / 3 \sqrt{3}, 2 i / 3 \sqrt{3}$. Let $R^{\prime}$ be 
another Riemann surface defined by the equation:

$$
y^{\prime 7}=x^{\prime 3}+x^{\prime}+p^{\prime}
$$

where $p^{\prime}$ is also a complex number different from $-2 i / 3 \sqrt{3}, 2 i / 3 \sqrt{3}$.

Then $(R, \sigma)$ is topologically equivalent to $\left(R^{\prime}, \sigma^{\prime}\right)$, where $\sigma, \sigma^{\prime}$ are naturally defined. In fact, for every such $p$ there exists a number $\alpha$ such that $y^{7}=x(x-1)(x-\alpha)$ is conformally equivalent to $y^{7}=x^{3}+x+p$. We see that the relation between $p$ and $\alpha$ is given by

$$
p^{2}=-\frac{(\alpha+1)^{2}(2 \alpha-1)^{2}(\alpha-2)^{2}}{27\left(\alpha^{2}-\alpha+1\right)^{3}}
$$

Now let $R: y^{7}=x^{3}+x+p$ and $R^{\prime}: y^{7}=x^{3}+x+p^{\prime}$ be equivalent for naturally defined automorphisms. Then we see that $p^{\prime}$ is equal to $\pm p$. Let $S^{\prime \prime}$ be the Riemann sphere punctured at $-2 i / 3 \sqrt{3}, 2 i / 3 \sqrt{3}$, and let $G$ be the group of automorphisms of the Riemann sphere generated by $\tau: P \rightarrow-P$. Here $P$ is the point of the sphere. Then there exists a one-to-one correspondence between the space $S^{\prime \prime} / G$ and the space $\Omega$. Here we see that $p=\infty$ corresponds to $\alpha=\frac{1+\sqrt{3} i}{2}$ and $\alpha=\frac{1-\sqrt{3} i}{2}$.

Finally, we shall apply our general theory to this case.

6.6. Let $S^{*}=\{x \mid x \in C\}$. Let $q$ be a generic point of $S^{*}$ over the field $k \supset Q(\zeta)$. We see that the field of moduli of $\mathscr{P}_{w\left(\lambda_{1}\right)} \subset k\left(q^{2}\right)$ (cf. [17]). Here $\lambda_{1}$ is a point corresponding to $q$. Then we can define a rational function $\psi$ such that

$$
\psi\left(q^{2}\right)=\varphi\left(w\left(\lambda_{1}\right)\right) .
$$

Therefore in the same way as $5.3-10$, we see that

$$
\psi\left(p^{2}\right)=\varphi(w(\lambda)),
$$

if $\lambda$ corresponds to $p$ in our sense. Here we notice that $p$ takes all values except for $p^{2}=-4 / 27$, i.e., $\phi$ is defined over all the points of the Riemann sphere punctured at $-4 / 27$ and infinity $\infty$. Here $\psi$ is a rational function of one variable, and so we may consider that $\psi$ is everywhere defined on the Riemann sphere.

On the other hand $\psi:$ the Riemann sphere $\rightarrow \widetilde{\mathfrak{B}}$ is one-to-one at the generic points (cf. [17]). Therefore since $\widetilde{\mathfrak{B}}$ can be regarded as the Riemann sphere, we have 


$$
\varphi\left(p^{2}\right)=\left(a p^{2}+b\right) /\left(c p^{2}+d\right)
$$

with constants $a, b, c$, and $d$.

Thus we have by $(6.6 .2)$

$$
p^{2}=(\varphi(w(\lambda)) d-b) /(-\varphi(w(\lambda)) c+a) .
$$

Therefore $p^{2}$ is a meromorphic function on $\Lambda$, and it has poles only at the points corresponding to $\alpha=(1 \pm \sqrt{3} i) / 2$.

6.7. THEOREM. Notations being as above,

$$
1 /\left(4+27 p^{2}\right)
$$

is a holomorphic function on the whole space $\Lambda$. And

$$
p^{2}=F(\lambda)
$$

is a meromorphic function in $\lambda$ on the generalized Teichmüller space $A$. Here $F$ is defined by (6.6.4), and cannot take the value $-4 / 27$, and has poles at $\lambda$ corresponding to $\alpha=(1 \pm \sqrt{3} i) / 2$ in the equation (6.1.1).

6.8. Remark. In $\widetilde{\mathfrak{B}}$, considered as the Riemann sphere, the point $-4 / 27$ is the unique point which does not correspond to any Riemann surfaces. It would be interesting to study the properties of the abelian variety corresponding to the point. We shall discuss this problem in another place.

\section{REFERENCES}

[1] Ahlfors, L. V., On quasiconformal mappings. J. Anal. Math., 3, 1-58 (1953-4).

[2] Ahlfors, L. V., The complex analytic structure of the space of closed Riemann surfaces. Analytic functions, Princeton Univ. Press., 1960, 45-60.

[ 3 ] Bers, L., Spaces of Riemann surfaces, Proc. Intern. Congr. Edinburgh, 1958.

[4] Bers, L., Quasiconformal Mappings and Teichmüller's Theorem. Analytic functions. Princeton Univ. Press., 89-119.

[5] Bers, L., Spaces of Riemann surfaces as bounded domains, Bull. Amer. Math. Soc., vol. 66 (1960), 98-103.

[6] Bers, L., Holomorphic differentials as functions of moduli, Bull. Amer. Math. Soc., vol. 67 (1961), 206-210.

[7] Eichler, M., Einfühlung in die Theorie der algebraischen Zahlen und Funktionen, Birkhäuser Verlag., 1963.

[ 8 ] Eichler, M., Eine Verallgemeinerung der Abelschen Integral. Math. Zeits. Bd., 67 (1957), 267-298.

[ 9 ] Hurwitz, A., Maht. Werke, Bd. I, Birkhäuser, Basel, 241-259, 321-390, 391-430.

[10] Igusa, J., Fiber systems of Jacobian varieties, Amer. J., of Math., 1956, 171-199. 
[11] Koizumi, S. and Shimura, G., On specializations of abelian varieties, Scientific Papers of the College of General Education, Univ. of Tokyo, 9 (1959), 187-211.

[12] Lewittes, J., Automorphisms of compact Riemann surfaces, Amer. J. of Math., (1963), 738-752.

[13] Lewittes, J., Invariant quadratic differentials, Bull. Amer. Math. Soc., 68 (1962), 320-322.

[14] Mangler, W., Die Klassen von topologischen Abbildungen einer geschlossenen FJäche auf sich, Math. Zeits. Bd 44 (1939), 513-554.

[15] Séminaire, H. Cartan, E. N. S., 1953-54, Théorie des fonctions de plusieurs variables.

[16] Shimura, G., On analytic families of polarized abelian varieties and automorphic functions, Ann. of Math., 78 (1963), 149-192.

[17] Shimura, G. On purely transcendental fields of automorphic functions of several variables, Osaka J. Math. I (1964), 1-14.

[18] Shimura, G., Moduli and fibre systems of abelian varieties. To appear.

[19] Teichmüller, O., Extremale quasikonforme Abbildungen und quadratische differentiale, Preuss-Akad. Math.-naturw. k1., 22, 1940.

[20] Tuji, R., On conformal mapping of a hyperelliptic Riemann surfaces onto itself, Kōdai Math. Sem. Rep., (1958), 127-136.

[21] Weil, A., Modules des surfaces de Riemann, Sem. Bourbaki May (1958).

\section{Chuo University}

